\title{
Linear stability analysis of wind turbine wakes performed on wind tunnel measurements
}

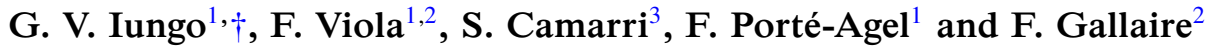 \\ ${ }^{1}$ Wind Engineering and Renewable Energy Laboratory, École Polytechnique Fédérale de Lausanne, \\ Lausanne, CH-1015, Switzerland \\ ${ }^{2}$ Laboratory of Fluid Mechanics and Instabilities, École Polytechnique Fédérale de Lausanne, Lausanne, \\ CH-1015, Switzerland \\ ${ }^{3}$ Department of Civil and Industrial Engineering, University of Pisa, Pisa, 56122, Italy
}

(Received 10 August 2012; revised 3 July 2013; accepted 25 October 2013; first published online 27 November 2013)

Wind tunnel measurements were performed for the wake produced by a three-bladed wind turbine immersed in uniform flow. These tests show the presence of a vorticity structure in the near-wake region mainly oriented along the streamwise direction, which is denoted as the hub vortex. The hub vortex is characterized by oscillations with frequencies lower than that connected to the rotational velocity of the rotor, which previous works have ascribed to wake meandering. This phenomenon consists of transversal oscillations of the wind turbine wake, which might be excited by the vortex shedding from the rotor disc acting as a bluff body. In this work, temporal and spatial linear stability analyses of a wind turbine wake are performed on a base flow obtained with time-averaged wind tunnel velocity measurements. This study shows that the low-frequency spectral component detected experimentally matches the most amplified frequency of the counter-winding single-helix mode downstream of the wind turbine. Then, simultaneous hot-wire measurements confirm the presence of a helicoidal unstable mode of the hub vortex with a streamwise wavenumber roughly equal to that predicted from the linear stability analysis.

Key words: absolute/convective instability, vortex instability, wakes/jets

\section{Introduction}

Wind turbine blade rotation produces a wake, which is the result of complex dynamics and interactions between different vorticity structures. The near wake is dominated by vortex roll-up, which is highly dependent on blade characteristics; its characterization is fundamental for optimization of rotor aerodynamics, to analyse vortex induced vibrations on blade structure and to reduce noise production typically connected to the shedding of the tip vortices. In the far wake, conversely, concentrated vorticity structures are no longer observed, and investigations have mainly focused on wake recovery and small-scale turbulent structures. Characterization of the far wake is fundamental for wind farm design and for evaluation of fatigue loads on downstream turbines. The connection between the vorticity structure formation in the near wake 
and the turbulence decay in the far wake is still an open question, as highlighted in Sørensen (2011).

The near wake of a wind turbine is characterized by the presence of counter-rotating helicoidal vorticity pairs shed from the tip and root of each blade. Furthermore, a system of trailing vortices is produced as a consequence of the varying circulation distribution along the blade span: see Sherry, Sheridan \& LoJacono (2010). Helicoidal tip vortices shed from a rotor have been characterized via different experimental approaches, e.g. particle image velocimetry (PIV) measurements in Whale et al. (1996) Massouh \& Dobrev (2007) and hot-wire anemometry in Chamorro \& PortéAgel (2009, 2010) and Zhang, Markfort \& Porté-Agel (2012). However, the only experimental work dealing with a detailed characterization of wind turbine near-wake vortex system, i.e. helicoidal tip and root vortices and trailing vortices, is that of Sherry et al. (2010), who performed PIV measurements in a water tunnel. Tip and root vortices are simultaneously shed from each blade, whereas at a very early stage trailing vortices are also detected. Tip vortices are rapidly convected downstream because they are located in a wake region with higher streamwise velocity with respect to the hub region, which is characterized by a strong velocity deficit. The helicoidal root vortices practically surround the wind turbine nacelle with a relatively small radius of curvature. This geometric feature and a reduced streamwise distance between adjacent vortex helices promote faster diffusion of the root vortices with respect to the tip vortices. In Sherry et al. (2010) root vortices are completely diffused at a downstream distance smaller than 0.5 rotor diameters $(d)$. Furthermore, interaction of root vortices with the nacelle boundary layer and wind turbine stem can further anticipate their diffusion. Diffusion of both root and tip vortices is found to be enhanced by increasing wind turbine tip speed ratio, which is due to the decreased helical pitch of the vortices, thus to the increased mutual induction between adjacent vortices (Widnall 1972).

Root and tip vortices have also been investigated in the near wake by means of direct numerical simulations of the Navier-Stokes equations in Ivanell et al. (2009). However, the main shortcoming of the numerical simulations of wind turbines consists in not resolving the boundary layer flow over the blades, and their induced loads are simulated through the actuator disc or actuator line models; thus, near-wake vorticity structures cannot be characterized with very high accuracy (Porté-Agel et al. 2011).

By moving downstream, root vortices are rapidly diffused, whereas a system of helicoidal tip vortices is still present; indeed, their signature in the velocity signals has been clearly detected by both numerical simulations (e.g. Lu \& Porté-Agel 2011) and experimental investigations (e.g. Medici \& Alfredsson 2006, Chamorro \& Porté-Agel 2009, 2010, and Zhang et al. 2012).

Regarding the central part of the wake, root vortices are found to diffuse rapidly in proximity to the wind turbine hub; however, a vorticity structure mainly oriented along the streamwise direction, which is denoted as the hub vortex, is still present several diameters downstream. The hub vortex is clearly visualized in the wake of a marine propeller by Felli, Camussi \& DiFelice (2011); regarding wind turbine wakes, the hub vortex is detected in Schito (2012) by means of smoke visualizations. With the PIV measurements carried out in Zhang et al. (2012), the hub vortex is observed up to a downstream distance of $1.5 \mathrm{~d}$.

Further downstream, several instabilities of wind turbine wakes are observed (see e.g. Medici \& Alfredsson 2008 and Felli et al. 2011), which can affect the wake recovery and increase fatigue loads for downstream wind turbines within a wind farm. In the so-called transitional region, the helicoidal tip vortices show an instability 
that is mainly driven by the mutual inductance between adjacent spirals. With the theoretical instability model of a helical vortex filament proposed by Widnall (1972), three instability modes are predicted: short-wave instability, long-wave instability and mutual-inductance instability. In Felli et al. (2011), via water tunnel visualizations of a marine propeller wake, all these three instability modes are found to be superimposed.

Joukowski (1912) considered a wake produced from a blade with a constant circulation along the blade span, which is composed of helicoidal tip vortices and root vortices. This vortex system is found to be unconditionally unstable, but it becomes stable if the trailing vortex sheet is also considered. Okulov \& Sørensen (2007) performed a linear stability analysis of helicoidal tip vortices, also including a vorticity field mimicking the presence of the hub vortex. These authors found that the stability of the tip vortices is strongly affected by the radial extent of the hub vortex and by its vorticity distribution. Furthermore, it is shown that the interaction between the hub vortex and the helicoidal tip vortices stabilizes the latter, otherwise they are always unstable and not affected by the number of blades and by the characteristics of the vorticity structures (see e.g. Levy \& Forsdyke 1928; Widnall 1972; Gupta \& Loewy 1974; Okulov 2004; Okulov \& Sørensen 2007). Ivanell et al. (2010) carried out a stability analysis of helicoidal tip vortices, with large eddy simulations of the Navier-Stokes equations using the actuator line model, and introducing small harmonic perturbations close to the blade tips. They found dispersive instabilities at several specific frequencies, and vortex pairing was also detected. Typically, helicoidal tip vortices diffuse more rapidly if immersed in a boundary layer flow; see e.g. Lu \& Porté-Agel (2011) and Porté-Agel et al. (2011). In Zhang et al. (2012) the helicoidal tip vortices are detected up to a distance of $2 d$.

Downstream with respect to the destabilization of the helicoidal tip vortices, Felli et al. (2011) found that the hub vortex also becomes unstable. By proceeding further downstream, the hub vortex starts oscillating sinusoidally, according to a spiral geometry, until breakdown occurs. This oscillation occurs with a frequency equal to that of the rotor, and is found to be independent of the number of blades. These authors hypothesize that the hub vortex instability can be excited by the perturbations produced by the instability of the helicoidal tip vortices. A low-frequency instability is also detected from wind tunnel tests of wind turbine models; see e.g. Medici \& Alfredsson (2006, 2008), Chamorro \& Porté-Agel (2010) and Zhang et al. (2012). In Medici \& Alfredsson (2008) this low-frequency instability, called wake meandering or wandering, is equal to 0.29 times the hub rotational frequency, and is ascribed to transversal oscillations of the wind turbine wake, which may be excited by the vortex shedding from the rotor disc in a similar way as for bluff bodies. This low frequency is found to decrease with increasing tip speed ratio of the turbine and with increasing thrust coefficient. In Chamorro \& Porté-Agel (2010) and Zhang et al. (2012), the low-frequency spectral component connected to wake meandering is equal to 0.34 times the hub rotational frequency, and it can be detected up to a downstream distance of $1.5 d$.

Further downstream, hub vortex breakdown occurs, as documented in Klein, Majda \& Damodaran (1995) and Ortega, Bristol \& Savas (2003). Sarpkaya (1971) and Felli et al. (2011) found that the hub vortex breakdown occurs as a double helix with the same rotation sense as the rotor.

In the far wake the helicoidal tip vortices and the hub vortex are completely diffused, and the streamwise velocity field shows a smooth quasi-Gaussian shape, with the location of the peak of the streamwise velocity deficit in correspondence to the hub height. Due to this wake feature, several analytical wake models have been 
proposed for the far wake (see e.g. Burton et al. 2001), which are mainly used for wind farm design. For cases of a wind turbine immersed in the boundary layer, the mean streamwise velocity component is skewed due to the oncoming boundary layer flow, but it recovers to an axisymmetric shape if the oncoming wind is subtracted, as shown in Chamorro \& Porté-Agel (2009). By moving downstream the wake gradually recovers with increasing wake width and decreasing velocity deficit.

In the present work linear stability analysis of wind turbine wakes is performed by using as a base flow velocity measurements averaged in time, which were carried out in a wind tunnel for a down-scaled wind turbine model. The main goal of this work is the physical characterization of the hub vortex low-frequency instability observed experimentally in the near wake. Both temporal and spatial linear stability analyses were performed; moreover, the Gaster transformation was also considered in order to produce a first approximation of the results then obtained through the spatial stability analysis. The stability properties of the experimental mean flow have been investigated in detail, and the results are used to reconstruct the mode associated with the instability of the hub vortex. The reconstructed mode has been successively assessed through further dedicated wind tunnel tests.

The work is organized as follows. Wind tunnel velocity measurements of wakes produced by a down-scaled wind turbine model with a uniform flow are described in $\S 2$. The numerical formulation of the linear stability analysis is presented in $\S 3$, while results of the temporal stability analysis are reported in $\S 4$. The spatial linear stability analysis is then described in $\S 5$. Concluding remarks are presented in $\S 6$.

\section{Wind tunnel measurements of wind turbine wakes}

\subsection{Set-up}

Velocity measurements of wind turbine wakes were performed in the boundary layer wind tunnel of the Wind Engineering and Renewable Energy Laboratory (WIRE) of the École Polytechnique Fédérale de Lausanne (EPFL), Switzerland. The facility is an open loop wind tunnel with an inlet providing a contraction with 4:1 area ratio. Several turbulence devices consisting of coarse meshes and honeycomb flow straighteners are used to increase flow quality. The wind tunnel consists of three different test sections: the section used for this work is the first one downstream of the inlet, which has a length of $28 \mathrm{~m}$, a width of $2.5 \mathrm{~m}$ and a height of $2 \mathrm{~m}$. This testing chamber provides an adjustable ceiling in order to eventually change the pressure gradient along the streamwise direction; for this work the pressure gradient was negligible $\left(\mathrm{d} C_{p} / \mathrm{d} x \approx-0.1 \% \mathrm{~m}^{-1}\right)$. The flow is produced by a single fan with a power of $90 \mathrm{~kW}$, producing a maximum velocity of $\sim 7 \mathrm{~m} \mathrm{~s}^{-1}$ with a minimum turbulence level of $2 \%$. A three-degrees-of-freedom traversing system allows probes to be positioned with an accuracy higher than $0.1 \mathrm{~mm}$.

The wind turbine down-scaled model used is a three-bladed GWS/EP-6030x3 anticlockwise. The rotor, with a diameter, $d$, of $152 \mathrm{~mm}$, is connected to a DC motor with a diameter of $10 \mathrm{~mm}$. The wind turbine model is mounted with a stem of height $127 \mathrm{~mm}$, which is in turn placed on a vertical support with a NACA 0018 crosssection and height of $500 \mathrm{~mm}$. This set-up enables the turbine to be placed outside the boundary layer, thus allowing it to have a uniform oncoming flow. A sketch of the experimental set-up is shown in figure 1. Typically for the tests considered here, the tip speed ratio of the turbine (TSR), which is the ratio between the speed of the blade tip and the oncoming velocity at hub height, $U_{\text {hub }}$, is 4.7 . The turbine's rotational 


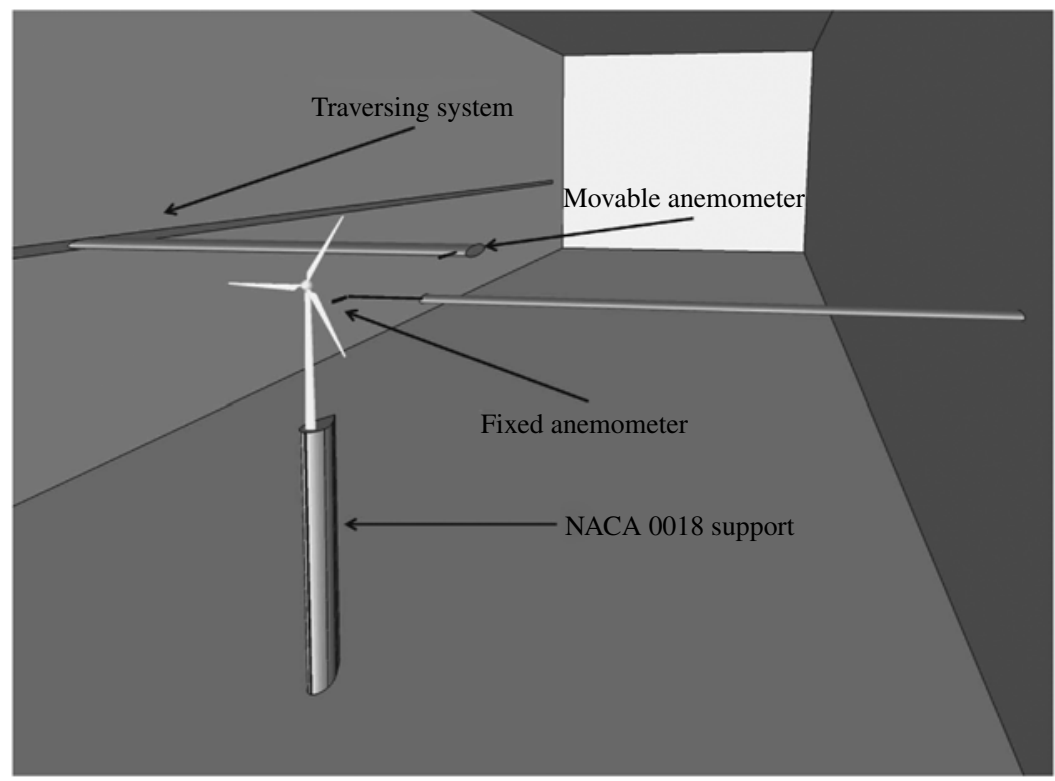

FIGURE 1. Sketch of the experimental set-up.

velocity was monitored through a laser tachometer manufactured by Monarch with an accuracy of $\sim 10$ r.p.m. For these tests the mean rotational frequency, $f_{\text {hub }}$, was $57 \mathrm{~Hz}$, with average fluctuations of $2.7 \%$ of the mean value. The velocity at hub height is $5.8 \mathrm{~m} \mathrm{~s}^{-1}$ and the turbulence level is equal to $7 \%$, which was obtained by removing one of the three turbulence screens located in the wind tunnel relaxation chamber. A Reynolds number of 60800 is obtained by considering $U_{h u b}$ as reference velocity, and the rotor diameter, $d$, as reference length.

The wind tunnel flow velocity was measured via two Pitot static tubes located near the entrance and end of the testing chamber; the Pitot tubes were in turn connected to two SETRA 239 transducers. Atmospheric pressure into the testing chamber was measured with a SETRA 276 transducer, and the static temperature was measured with a Pt100 Eliwell SN206000. All the analogue signals were acquired with three NI PXI-6143 data acquisition systems.

Measurements of three components of the velocity were performed with a customized Cobra probe produced by Turbulent Flow Instrumentation, which is a four-hole pressure probe. This probe, with an external diameter of $1.5 \mathrm{~mm}$, can measure velocity fluctuations characterized by frequencies lower than $300 \mathrm{~Hz}$. The maximum admissible angle between the probe stem and the wind direction is $\sim 45^{\circ}$. This miniaturized version of the Cobra probe allows us to obtain accuracy higher than $2 \%$ in the velocity module and $\sim 1^{\circ}$ for pitch and yaw angles. Cobra measurements were typically carried out with a sampling frequency of $5 \mathrm{kHz}$ and a number of samples equal to $2^{19}$.

Wake measurements were also performed with hot-wire anemometers, both singlecomponent (DANTEC 55P11) or cross-wire probes (DANTEC 55P61). For the simultaneous hot-wire measurements presented in $\$ 5$, hot-wire probes with rightangled prongs and with the sensor perpendicular to the probe axis were used (DANTEC 55P14). These probes were introduced from the wake side in order 

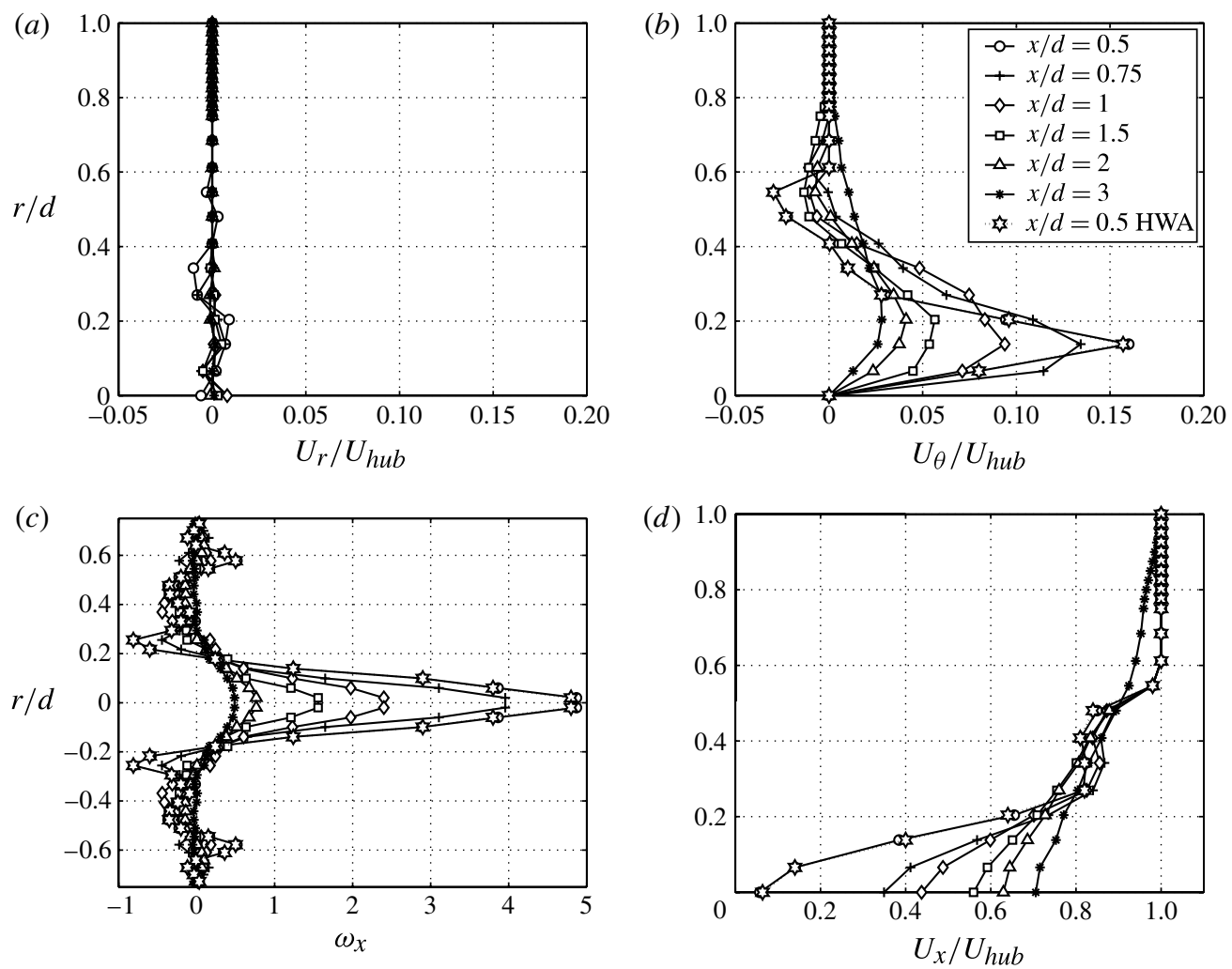

FIGURE 2. Mean velocity field of the wind turbine wake: $(a)$ radial velocity, $U_{r} ;(b)$ azimuthal velocity, $U_{\theta} ;(c)$ non-dimensional axial vorticity, $\omega_{x} ;(d)$ axial velocity, $U_{x}$. These data are obtained with the Cobra probe, except for $x / d=0.5$ HWA, obtained by hot-wire anemometry.

to reduce intrusiveness of the measuring system. The hot-wire anemometers were connected to an A.A. Lab Systems AN-1003. Their calibration was performed by setting the probes on a StreamLine Pro Automatic Calibrator manufactured by Dantec, which guarantees flow accuracy higher than $1 \%$. Hot-wire measurements were typically carried out with a sampling frequency of $20 \mathrm{kHz}$ and a number of samples equal to $2^{21}$. Statistics of the hot-wire measurements were generally in good agreement with those performed with the Cobra probe (see figure 2); however, their higher-frequency resolution allowed more accurate spectral analyses to be performed.

The reference frame used has its origin placed just behind the hub of the wind turbine, with the $x$-axis corresponding to the streamwise direction, positive pointing downstream. The $z$-axis is along the vertical direction, positive from the bottom towards the top, while the $y$-axis is along the spanwise direction oriented so as to produce a right-handed Cartesian coordinate system.

Velocity measurements were performed from a downstream distance of $0.125 d$ up to $20 \mathrm{~d}$. For each downstream location considered, two-dimensional maps were measured with an area of $1.5 d \times 1.5 d$ with a spacing between adjacent points of $\sim 0.025 d$. Moreover, measurements over the horizontal and vertical planes passing through the hub location were performed with a higher resolution along the streamwise direction. 


\subsection{Velocity measurements}

As already proven in previous experimental and numerical works (Alfredsson \& Dahlberg 1979; Vermeer, Sørensen \& Crespo 2003; Medici \& Alfredsson 2006) and confirmed by the present experimental campaign, the wake velocity field produced by a wind turbine immersed in a uniform flow can be considered to be axisymmetric with respect to the axis passing through the hub location and directed along the oncoming wind direction, i.e. the $x$-axis. Two-dimensional measurements performed over transversal planes located at different downstream locations, not reported here for the sake of brevity, confirmed that velocity profiles are invariant for different azimuthal orientations of the traverses. A small wake produced by the wind turbine stem is detected in the very near wake; however, it is completely recovered at the downstream locations considered for this investigation. Moreover, the radial velocity is also found to be practically negligible, as shown in figure 2(a). Therefore, the wake flow can be completely characterized through the axial and azimuthal velocities as a function of the radial and streamwise positions. In figure 2 the radial velocity, $U_{r}$, the mean azimuthal velocity, $U_{\theta}$, the non-dimensional axial vorticity, $\omega_{x}$, and the axial velocity, $U_{x}$, are shown as a function of the radial position and for several downstream locations considered for the linear stability analysis. For the sake of brevity, only the mean velocity obtained via the Cobra probe measurements is presented; however, the velocity obtained from the two component hot-wire anemometry is practically equal, i.e. with differences lower than $1 \%$ for each location, as shown in figure 2 for the measurements carried out at the downstream location $x / d=0.5$.

In figure $2(b)$ significant peaks of the azimuthal velocity are detected for radial positions $r / d \approx 0.15$, which are connected to the rotational velocity induced by the hub vortex, which is a vorticity structure mainly oriented along the streamwise direction. Indeed, a dominant peak of the axial vorticity is typically detected in figure $2(c)$ corresponding to the wake centre, and it is shielded by a ring-like negative vorticity structure, which is the result of the time-averaging of the helicoidal tip vortices. The hub vortex produced by a rotor has already been detected in the near wake by several experimental works, e.g. Felli et al. (2011) and Zhang et al. (2012). The azimuthal velocity connected to the hub vortex and its related axial vorticity are rapidly reduced by moving downstream, and the hub vortex can be considered as completely diffused for downstream distances larger than $4 d$. Secondary peaks of the azimuthal velocity are also observed for radial positions approximately equal to $r / d \approx 0.5$, which are related to the presence of the helicoidal tip vortices. Further, the azimuthal velocity connected to the tip vortices is decreased by moving downstream, and at $x / d=4$ it is practically diffused.

In figure $2(d)$ the axial velocity field shows a significant deficit in correspondence to the wake centre, which gradually recovers by moving downstream, while wake width increases. A velocity deficit connected to the presence of the helicoidal tip vortices is also observed in the near wake at $r / d \approx 0.5$, but it disappears as the helicoidal tip vortices diffuse.

Spectral analysis of the velocity signals acquired in the wind turbine wake clearly shows the presence of two main spectral components, as presented in figure 3: a low frequency, which is typically detected in correspondence to the wake core within a radial distance of about $r / d \approx 0.2$, and a high frequency related to the shedding of the tip vortices. In the present investigation the high frequency connected to the tip vortex shedding is $171 \mathrm{~Hz}$, which is equal to three times the rotational frequency of the turbine (a three-bladed model was tested). On the other hand, the spectral energy detected in the central part of the wake has a frequency of $\sim 20 \mathrm{~Hz}$. This frequency, 


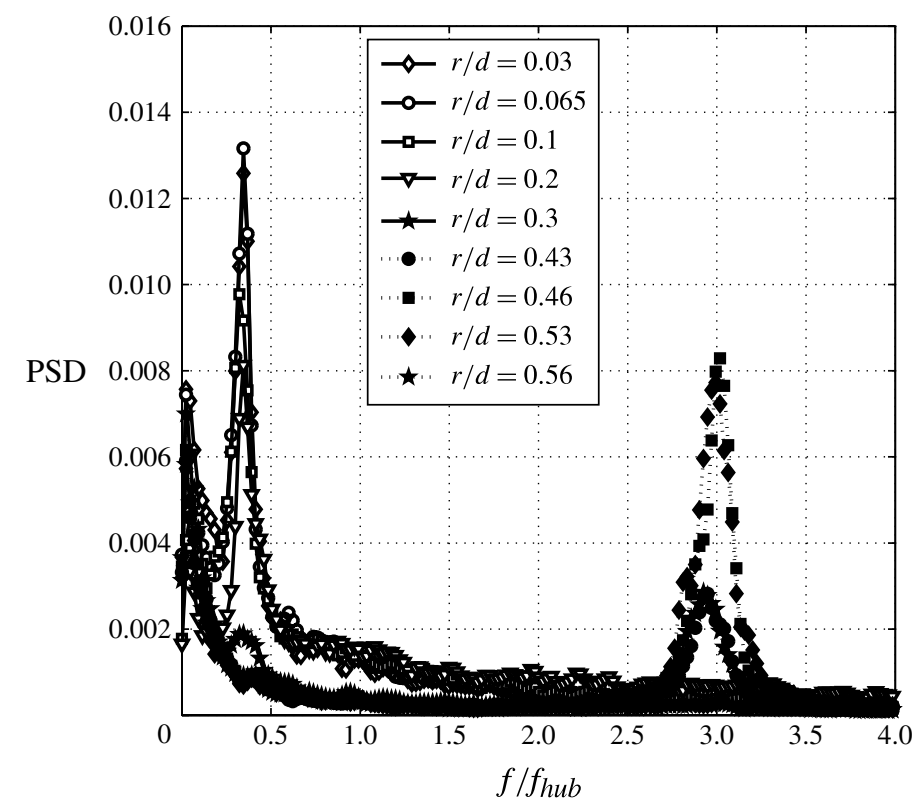

FIGURE 3. Fourier power spectral density of the velocity signals acquired at $x / d=0.5$, $z / d=0$, and different radial locations.

which could be connected to the hub vortex oscillations, is equal to 0.34 times the hub rotational frequency, which is in good agreement with results of previous works, e.g. Medici \& Alfredsson (2008), Chamorro \& Porté-Agel (2010) and Zhang et al. (2012). This low-frequency spectral contribution corresponds to a non-dimensional frequency of $\omega=2 \pi f d / U_{h u b}=3.18$. In figure 4 the average power spectral density obtained from two-dimensional measurements performed in the wake core within a radial distance of $r / d=0.2$ is reported for several downstream locations. It is shown that the spectral energy connected to this low-frequency component increases by moving downstream up to $x / d=1$, then further downstream its energy starts to decrease.

Tests were also performed by setting different values of the tip speed ratio (TSR). Spectral analysis of the velocity signals, briefly summarized in table 1, shows that both the frequency connected to the helicoidal tip vortices and that related to the hub vortex vary roughly linearly with the rotational frequency of the rotor, $f_{h u b}$. This result indicates that the dynamics of these vorticity structures are dependent on the wake swirl, and confirms an outcome presented in Felli et al. (2011), where an invariant hub vortex frequency was detected by changing the tip vortex frequency by means of a different number of rotor blades.

\section{Linear stability analysis: formulation of the numerical problem}

In this section the classical local linear stability theory of shear flows is presented, which is related to the development in space and time of infinitesimal perturbations on a given base flow. The base flow is assumed to be quasi-parallel and slowly evolving along the streamwise direction. Flow fluctuations are typically decomposed into elementary instability waves of axial and azimuthal wavenumbers $k$ and $m$, 


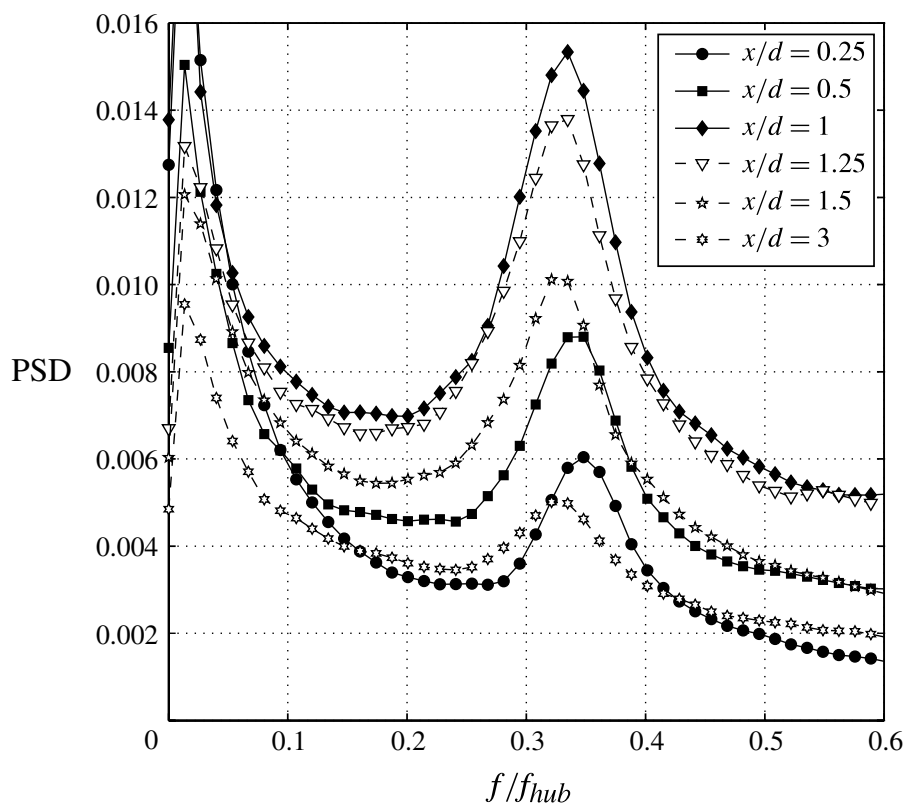

FIGURE 4. Average power spectral density obtained from two-dimensional velocity measurements performed within the wake core with a radius of $r / d=0.2$, and evaluated for different downstream locations.

$\begin{array}{lccccc}\text { Case } & U_{\text {hub }}\left(\mathrm{m} \mathrm{s}^{-1}\right) & \text { TSR } & f_{\text {hub }}(\mathrm{Hz}) & f_{\text {tip vortices }}(\mathrm{Hz}) & f_{\text {hub vortex }}(\mathrm{Hz}) \\ 1 & 5.8 & 7.6 & 92 & 276 & 31.5 \\ 2 & 5.8 & 5.3 & 65 & 195 & 21.5 \\ 3 & 5.8 & 4.7 & 57 & 171 & 20 \\ 4 & 5.8 & 3.2 & 39 & 118 & 17\end{array}$

TABLE 1. Spectral analysis for the tests performed with different TSR values.

respectively, and frequency $\omega$. The perturbations satisfy an ordinary differential equation of Orr-Sommerfeld type. Enforcement of appropriate boundary conditions then leads to an eigenvalue problem, whereby eigenfunction solutions for the perturbations exist only if $k, m$ and $\omega$ satisfy a dispersion relation of the form

$$
D[k, m, \omega]=0 .
$$

Temporal modes refer to cases where the complex frequency $\omega$ is determined as a function of real wavenumbers $k$ and $m$. Conversely, spatial branches are obtained by solving the dispersion relation with complex wavenumbers $k$, whereas $m$ and $\omega$ are real numbers.

The base flow used for the linear stability analysis was obtained by averaging in time the above-mentioned wind tunnel velocity measurements, as was done by Oberleithner et al. (2011) to study the breakdown of swirling jets. Although the mean flow does not represent an equilibrium point for the system, accurate results can be obtained by a stability analysis, as proven in Barkley (2006), Leontini, Thompson \& Hourigan (2010) and Camarri, Fallenius \& Fransson (2013), for example. The 
relevance of considering the stability of the time-averaged flow was assessed by Sipp \& Lebedev (2007) theoretically close to onset of instability. In the presence of background turbulence, despite the lack of a solid theoretical framework, stability analyses of mean flows were observed to accurately compare with experimental observations by Monkewitz (1988) and Meliga, Sipp \& Chomaz (2009), for instance.

The wind turbine wake flow is practically axisymmetric and has a negligible radial velocity: see $\S 2$. Moreover, according to the local nature of the present stability analysis, for each section the flow is considered to be invariant for translations along the $x$-axis. Let us consider the base flow expressed in cylindrical coordinates:

$$
U=\left(\begin{array}{c}
U_{r} \\
U_{\theta} \\
U_{x} \\
P
\end{array}\right)=\left(\begin{array}{c}
0 \\
r \Omega(r) \\
W(r) \\
P(r)
\end{array}\right),
$$

where $U_{x}$ is the streamwise velocity, $U_{\theta}$ is the azimuthal velocity, $U_{r}$ is the radial velocity and $P$ is the pressure field. The radial velocity, $U_{r}$, is identically null, while the azimuthal velocity, $U_{\theta}$, is proportional to the the angular velocity, $\Omega$.

According to the linear stability analysis, the evolution of small perturbations can be described by the Navier-Stokes equations linearized on the base flow. Let us consider the normal modes expansion of the perturbation:

$$
\boldsymbol{u}(r, \theta, x, t)=\hat{\boldsymbol{u}}(r, t) \mathrm{e}^{\mathrm{i}(m \theta+k x)}
$$

where $k$ is the axial wavenumber, $m$ is the azimuthal wavenumber and $\mathrm{i}$ is the imaginary unit. The expansion in normal modes imposes a helicoidal symmetry on the perturbation, allowing us to investigate each Fourier component separately by solving the corresponding one-dimensional problem for each pair $(k, m)$. The continuity equation and the momentum equations in cylindrical coordinates are

$$
\begin{gathered}
\frac{1}{r} \frac{\partial\left(r u_{r}\right)}{\partial r}+\frac{\mathrm{i} m}{r} u_{\theta}+\mathrm{i} k u_{x}=0 \\
\left(\frac{\partial}{\partial t}+\Gamma_{m, k}\right) u_{r}-2 \Omega u_{\theta}=-\frac{\partial p}{\partial r}+\frac{1}{R e}\left(\Delta_{m, k} u_{r}-\frac{u_{r}}{r^{2}}-\frac{2 \mathrm{i} m}{r^{2}} u_{\theta}\right) \\
\left(\frac{\partial}{\partial t}+\Gamma_{m, k}\right) u_{\theta}+\left(r \frac{\partial \Omega}{\partial r}+2 \Omega\right) u_{r}=-\frac{\mathrm{i} m}{r} p+\frac{1}{R e}\left(\Delta_{m, k} u_{\theta}-\frac{u_{\theta}}{r^{2}}+\frac{2 \mathrm{i} m}{r^{2}} u_{r}\right) \\
\left(\frac{\partial}{\partial t}+\Gamma_{m, k}\right) u_{x}+\frac{\partial W}{\partial r} u_{r}=-i k p+\frac{1}{R e}\left(\Delta_{m, k} u_{x}\right)
\end{gathered}
$$

where $\Gamma_{m, k}$ and $\Delta_{m, k}$ are expressed as follows:

$$
\begin{gathered}
\Gamma_{m, k}(r)=\mathrm{i} m \Omega(r)+\mathrm{i} k W(r), \\
\Delta_{m, k}=\frac{1}{r} \frac{\partial}{\partial r}\left(r \frac{\partial}{\partial r}\right)-\frac{m^{2}}{r^{2}}-k^{2} .
\end{gathered}
$$

In order to solve numerically the equation system (3.4), a reduced formulation is obtained by using the variable $\boldsymbol{v}=\left(u_{r}, u_{\theta}\right)^{\mathrm{T}}$,

$$
\boldsymbol{F}(\boldsymbol{v})=L \frac{\partial v}{\partial t}+C \boldsymbol{v}-\frac{1}{R e} \boldsymbol{D} v=0,
$$


where the operators are defined as follows:

$$
\begin{aligned}
\boldsymbol{L} & =\left(\begin{array}{ll}
1 & 0 \\
0 & 1
\end{array}\right)+\boldsymbol{b}_{1} \boldsymbol{b}_{2}^{T} \\
\boldsymbol{C} & =\left(\begin{array}{cc}
\Gamma_{m, k} & -2 \Omega \\
\frac{\partial \Omega}{\partial r}+2 \Omega & \Gamma_{m, k}
\end{array}\right)+\boldsymbol{b}_{1} \Gamma_{m, k} \boldsymbol{b}_{2}^{T}-\boldsymbol{b}_{1}\left(\frac{\partial W}{\partial r}, 0\right) \\
\boldsymbol{D} & =\left(\begin{array}{cc}
\Delta_{m, k}-\frac{1}{r^{2}} & -\frac{2 \mathrm{i} m}{r^{2}} \\
\frac{2 \mathrm{i} m}{r^{2}} & \Delta_{m, k}-\frac{1}{r^{2}}
\end{array}\right)+\boldsymbol{b}_{1} \Delta_{m, k} \boldsymbol{b}_{2}^{T}, \\
\boldsymbol{b}_{1}^{T} & =\frac{1}{\mathrm{i} k}\left(\frac{\partial}{\partial r}, \frac{\mathrm{i} m}{r}\right), \\
\boldsymbol{b}_{2}^{T} & =\frac{1}{\mathrm{i} k}\left(\frac{\partial}{\partial r}+\frac{1}{r}, \frac{\mathrm{i} m}{r}\right) .
\end{aligned}
$$

For the stability analysis, a code based on a Chebyshev spectral collocation method is used: see Antkowiak (2005) for more details. The Gauss-Lobatto-Chebyshev collocation grid is mapped into the infinite physical space through algebraic map $r=L \zeta / \sqrt{1-\zeta^{2}}$ (Canuto et al. 1993), where the parameter $L$ is set in order to fix the second-to-last and the second point to $r_{\max }$ and $-r_{\max }$, respectively. Moreover, taking into account the parity of the functions in the expression of the derivatives (Kerswell \& Davey 1996), and imposing the boundary conditions at infinity, the equations are discretized in the finite domain $\left.] 0, r_{\max }\right]$. The computational sensitivity related to the number of collocation points, $N$, and the size of the physical domain was investigated, but for the sake of brevity these results are not reported here. The selected settings with $N=120$ and $r_{\max }=50$ are seen to provide the convergence of the most unstable eigenvalue with five-digit accuracy, which is deemed to be sufficient for the present purposes. For the stability analysis the same Reynolds number was used as in the experimental measurements, i.e. 60800 ; indeed, the equations used were made nondimensional by using $U_{h u b}$ and $d$ as reference dimensions.

The experimental data were fitted with spline functions in order to produce an adequate spatially resolved base flow for the stability analysis. Different fitting methods were tested and they were observed not to affect the results of the stability analysis.

\section{Temporal stability analysis}

Temporal stability analysis allows the investigation of the temporal evolution of disturbances on a base flow within a volume large enough to include the complete disturbance: see Schmid \& Henningson (2001). After a transient period the evolution of the perturbations is dominated by the less damped mode, which is in the form $\mathrm{e}^{-\mathrm{i} \omega t}$ with $\omega \in \mathbb{C}$. In particular, if $\omega_{i}=\operatorname{Im}(\omega)>0$, the corresponding mode is unstable with a growth rate equal to $\omega_{i}$, while $\omega_{r}=\operatorname{Re}(\omega)$ is the frequency related to the propagating harmonic mode (Lighthill 1978). Considering harmonic perturbations in time, equation (3.6) becomes

$$
\boldsymbol{F}_{\omega}(\boldsymbol{v})=-\omega \mathrm{i} L v+C v-\frac{1}{R e} \boldsymbol{D} v=0
$$




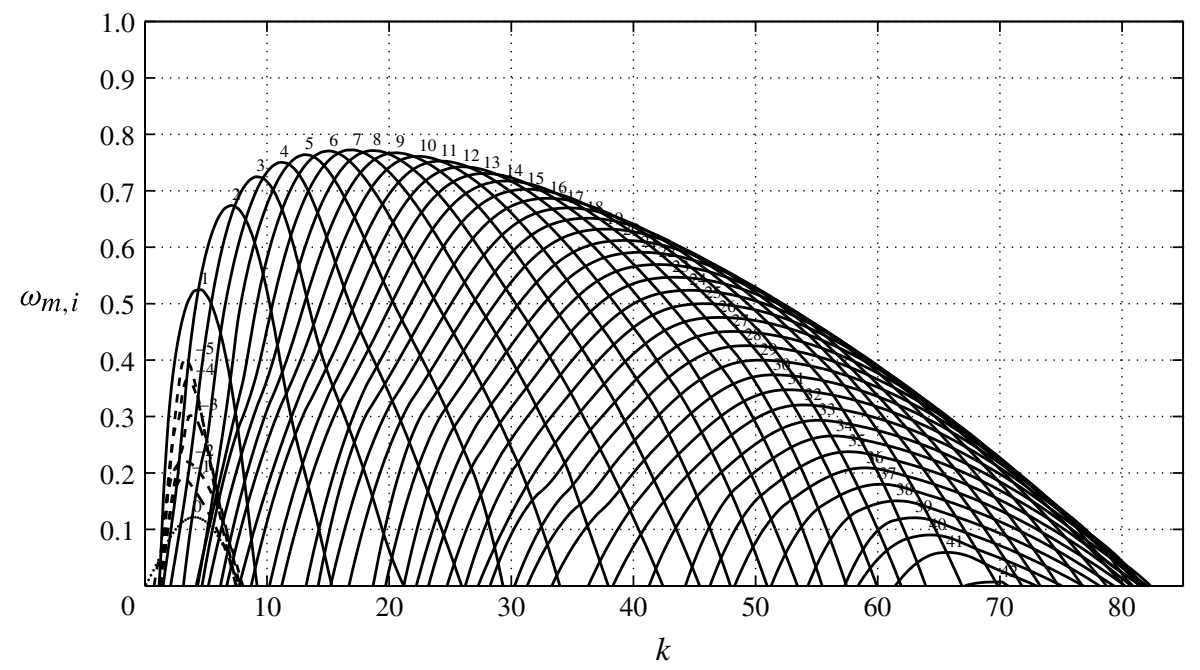

FIGURE 5. Growth rates $\left(\omega_{m, i}\right)$ of the unstable modes as a function of the axial wavenumber $k$ for the downstream location $x / d=1$.

In order to carry out the temporal stability analysis, (4.1) is integrated with $k \in \mathbb{R}$, $m \in \mathbb{R}$ and $\omega \in \mathbb{C}$. By considering normal mode expansion, as in (3.3), positive values of $m$ correspond to modes winding in the opposite direction with respect to the rotation inferred by the hub vortex; see Gallaire \& Chomaz (2003) for a more detailed discussion. For each pair $(k, m)$, solving (4.1) is equivalent to solving the following generalized eigenvalue problem:

$$
\boldsymbol{A}(k) \boldsymbol{v}=\omega \boldsymbol{B}(k) \boldsymbol{v}
$$

where, referring to (4.1),

$$
\boldsymbol{A}(k)=\boldsymbol{C}-\frac{1}{\operatorname{Re} \boldsymbol{D}} \quad \boldsymbol{B}(k)=\mathrm{i} \boldsymbol{L} .
$$

The growth rates $\omega_{m, i}(k)$ of the unstable modes calculated as a function of the axial wavenumber, $k$, and different $m$ are shown in figure 5. For the downstream location $x / d=1$ considered, the modes are unstable for $k \leqslant 82$ and for values of $m$ up to 42 . The maximum growth rate connected to the most unstable mode is in good agreement with the value predicted via the Leibovich-Stewartson instability criterion. The latter is a generalization of the Rayleigh criterion and provides a necessary instability condition for a rotating inviscid vortex in the presence of axial flow (Leibovich \& Stewartson 1983). As for the above-mentioned criterion, the vortex is unstable when the following sufficient condition is satisfied:

$$
\omega_{i}^{2}=\frac{2 U_{\theta}\left(r \mathrm{D} U_{\theta}-U_{\theta}\right)\left(U_{\theta}^{2} / r^{2}-\left(\mathrm{D} U_{\theta}\right)^{2}-\left(\mathrm{D} U_{x}\right)^{2}\right)}{\left(r \mathrm{D} U_{\theta}-U_{\theta}\right)^{2}+r^{2}\left(\mathrm{D} U_{x}\right)^{2}}>0,
$$

where $\mathrm{D}$ is the spatial first derivative along the radial direction. Moreover, when the inequality (4.4) is satisfied for a certain value of $r$, that relation provides the square of the growth rate of the most unstable mode corresponding to an eigenfunction with a certain characteristic size, $r$ : see Gallaire \& Chomaz (2003) for details. Figure 6 shows that the Leibovich-Stewartson instability criterion, evaluated for the 


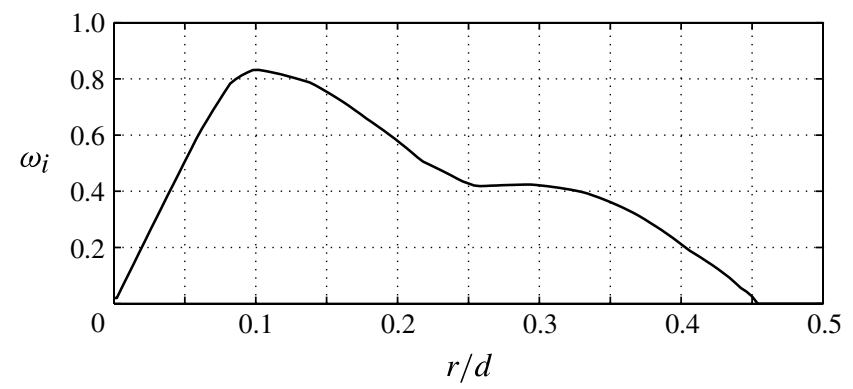

FIGURE 6. Growth rate predicted via the Leibovich-Stewartson instability criterion for the measurements carried out at $x / d=1$.

measurements carried out at $x / d=1$, predicts instability for every radial position with the highest values in proximity to the azimuthal velocity peak connected to the hub vortex (see figure $2 a$ ). The growth rate of the most unstable mode predicted via the Leibovich-Stewartson criterion is $\sim 0.8$, which confirms the result obtained with the temporal stability analysis shown in figure 5.

The main goal of the present work is the characterization of the low-frequency spectral component detected through the wind tunnel velocity measurements carried out into the core of the wind turbine wake, which are interpreted as the effect of an instability of the hub vortex. To this end, in figures 7 and 8 the growth rate $\omega_{i}$ is plotted as a function of the non-dimensional frequency, $\omega_{r}$, for the different downstream locations analysed. In these figures the non-dimensional frequency related to the above-mentioned low-frequency instability is indicated by a vertical dashed line corresponding to the value 3.18, obtained from the spectral analysis of the velocity signals (see figures 3 and 4).

Starting from the location $x / d=0.5$, the selected unstable mode corresponding to the non-dimensional frequency related to the hub vortex instability is $m=1$, i.e. a counter-winding single-helix mode. However, the unstable mode $m=1$ is not the one with the highest growth rate overall. For the unstable high-frequency modes, the diffusive contribution of the turbulent fluctuations, which is neglected in the present stability analysis, is expected to damp them significantly, in particular as $m$ increases, so as to change the picture given in figure 5. Indeed, one of the simplest ways to take into account this effect in the stability analysis might be to include an eddy viscosity turbulence model in (3.4). Assuming, in its simplest approximation, a constant eddy viscosity model would be equivalent to reducing the effective Reynolds number of the stability analysis and, in turn, this would decrease the growth rate of the unstable modes depending on $m$; indeed, the viscous terms scale as $m^{2}$. This aspect, which is delicate and mentioned here only very briefly, is the object of ongoing investigations by the authors, and at present the interpretation provided here is confirmed by preliminary results.

By moving downstream, the scenario remains practically unchanged with a general reduction of the growth rate for all unstable modes. However, at the downstream position of $x / d=2.5$, the non-dimensional frequency corresponding to the hub vortex instability starts selecting the mode $m=2$, i.e. the counter-winding double-helix mode. For the location $x / d=4$ the mode $m=4$ is selected. In order to provide a physical interpretation of this stability analysis, several factors should be considered. First, the growth rates generally decrease by moving downstream, as shown in figure 9 for the 


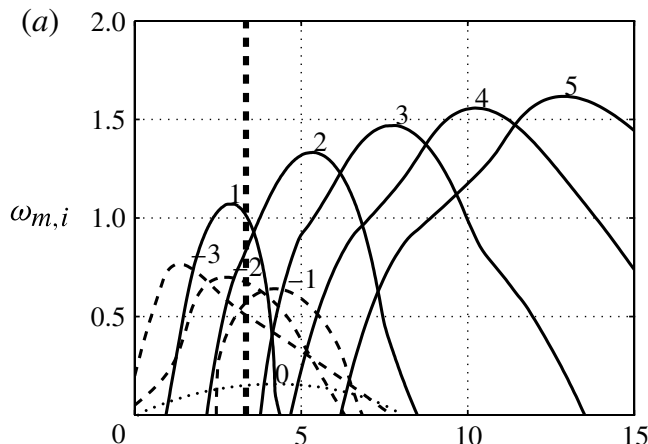

(b)

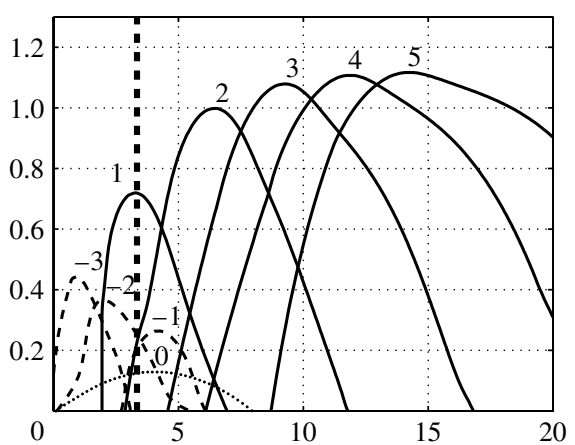

(c)

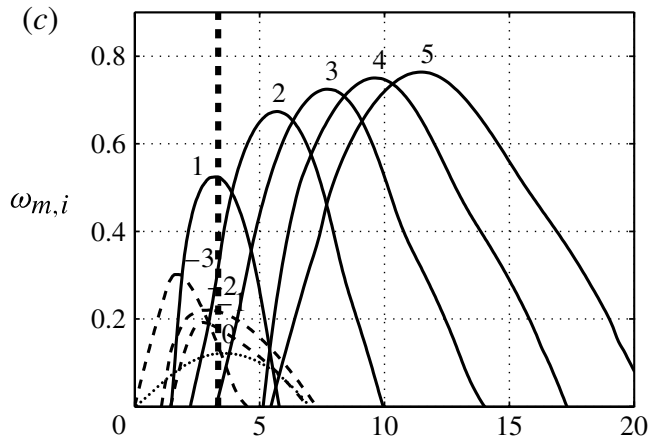

(e)

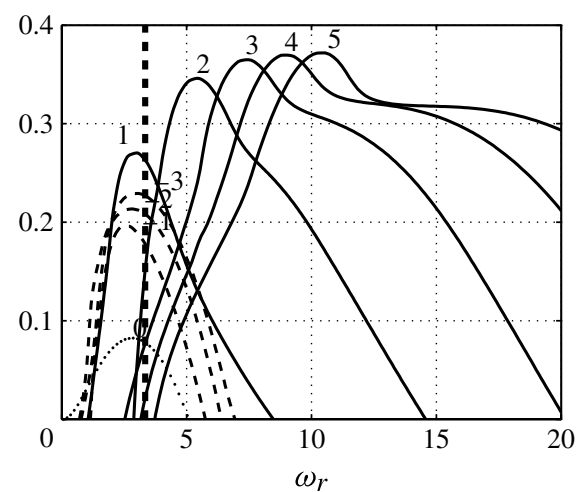

$(d)$

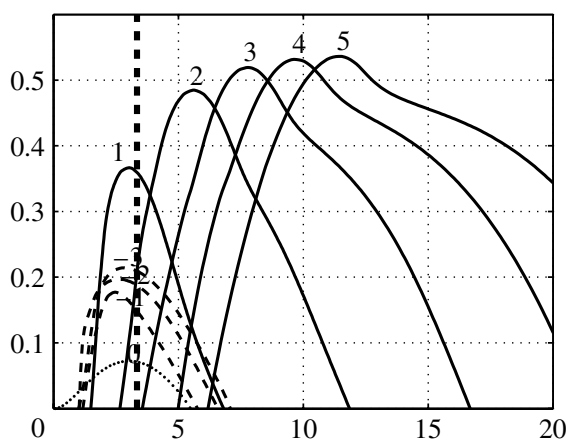

$(f)$

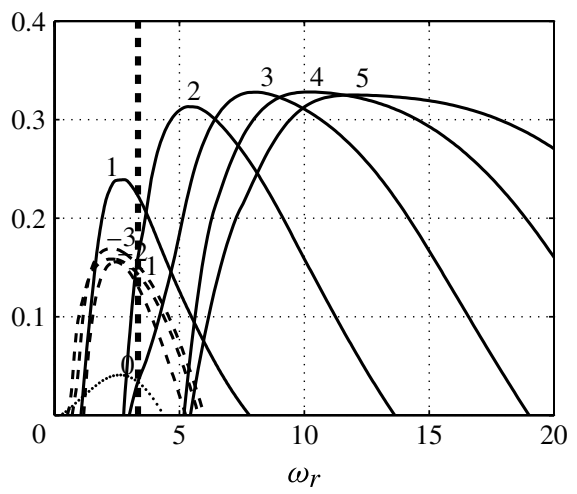

FIGURE 7. Growth rate as a function of the non-dimensional frequency for downstream locations from $x / d=0.5$ up to $x / d=1.75$. The vertical dashed line corresponds to the non-dimensional frequency measured experimentally and related to the hub vortex instability: (a) $x / d=0.5 ;$ (b) $x / d=0.75 ;(c) x / d=1 ;(d) x / d=1.25 ;(e) x / d=1.5 ;(f) x / d=1.75$.

mode $m=1$. Moreover, the hub vortex diffuses rapidly by moving downstream, as shown by the experimental measurements in figure 2 . Therefore, the unstable mode with $m=1$ should dominate because it is the mode selected through the hub vortex instability frequency $\left(\omega_{r}=3.18\right)$ in the very near wake, which is a wake region where the hub vortex has a significant vorticity.

The temporal analysis suggests that in the near wake the hub vortex is characterized by a single-helix counter-winding instability, i.e. $m=1$. Furthermore, by considering that $\omega_{r}$ is positive, the helicoidal unstable mode is rotating in the same direction as the 


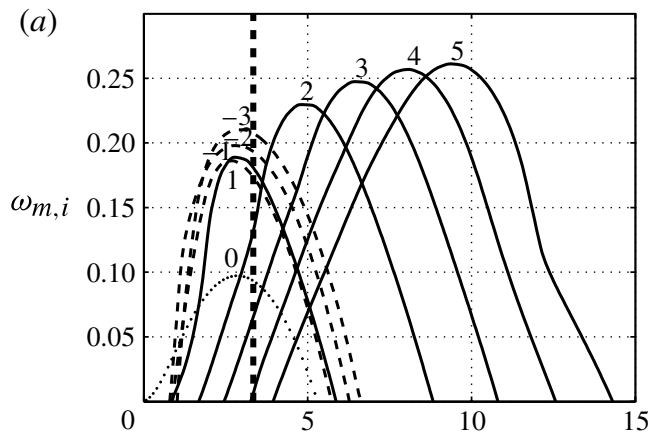

(b)

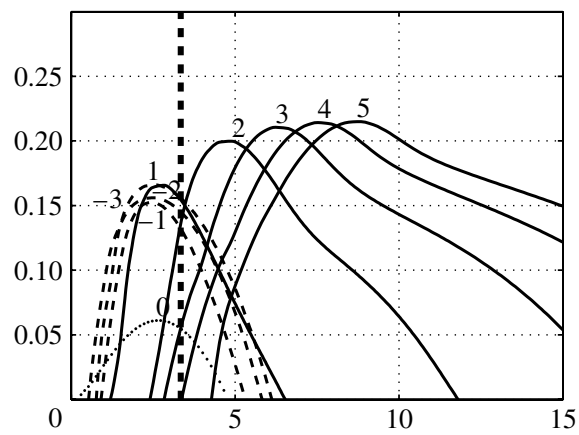

(c)

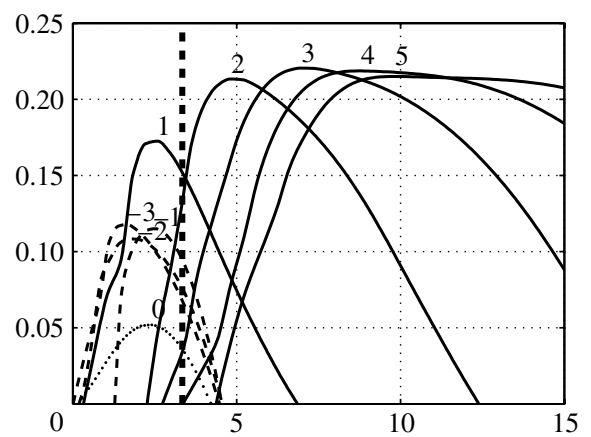

(d)

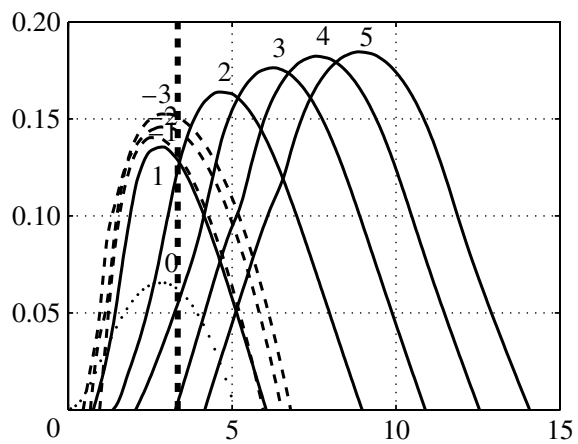

(e)
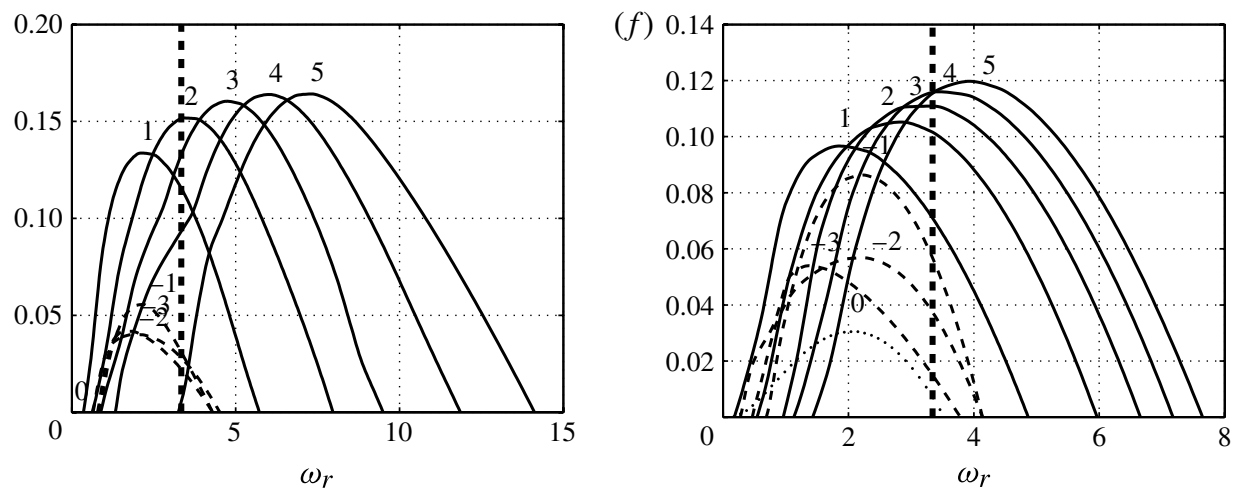

FIGURE 8. Growth rate as a function of the non-dimensional frequency for downstream locations from $x / d=2$ up to $x / d=4$. The vertical dashed line corresponds to the nondimensional frequency measured experimentally and related to the hub vortex instability: (a) $x / d=2 ;(b) x / d=2.25 ;(c) x / d=2.5 ;(d) x / d=2.75 ;(e) x / d=3 ;(f) x / d=4$.

hub vortex. By moving downstream the hub vortex diffuses, and unstable modes with higher values of $m$ could be selected, even with decreasing growth rates. This mode competition will be better investigated in the following section by means of the spatial stability analysis.

\section{Spatial stability analysis and characterization of the hub vortex instability}

This section is devoted to the spatial stability analysis of disturbances generated at a fixed position within a wind turbine wake and evolving downstream. In the 


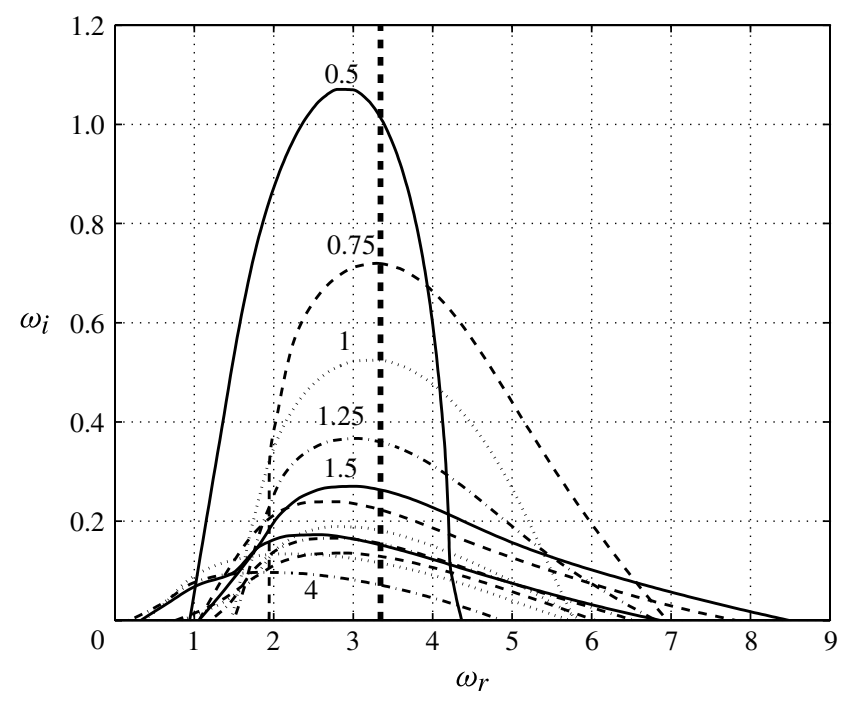

FIGURE 9. Growth rate as a function of the non-dimensional frequency for the mode $m=1$ and different downstream locations. The vertical dashed line corresponds to the nondimensional frequency measured experimentally and related to the hub vortex instability.

temporal setting of $\S 4$, the mode $m=1$ could not be singled out, although it was seen to 'resonate' well at the frequency observed in the experiments. In order to better elucidate this mode selection, a spatial stability analysis is more suitable, since it is adapted to convectively unstable flows submitted to incoming noise. These flows are known to act as selective amplifiers. The weakly non-parallel spatial stability theory is the preferred analysis tool to better understand this selection.

Note, however, that a spatial stability analysis is only justified if the flow is convectively unstable. We have therefore checked that the flow is not absolutely unstable for $x / d>0.5$ where the present analysis applies. The presented measurements do not allow us to determine the nature of the instability closer to the hub, where a backflow region could be expected. We can therefore only speculate on the existence of a sufficiently extended pocket of absolutely unstable flow in the very near-wake region as the origin of the sharp frequency selection observed at $x / d=0.5$ and beyond, through the development of a self-sustained steep nonlinear global mode (Pier \& Huerre 2001; Chomaz 2005). While it cannot elucidate the detailed origin of the spectral signature, the spatial stability analysis of the convective region is perfectly valid: it will be seen to be very useful in characterizing the spatial structure of the mode.

The numerical formulation of the spatial stability analysis is analogous to that of the temporal analysis (see (4.1)), with the exception that a complex streamwise wavenumber, $k$, and a real frequency, $\omega$, are now considered. Therefore, the spatial stability analysis consists of a nonlinear polynomial eigenvalue problem, as follows:

$$
A_{0}(\omega) \boldsymbol{v}+k A_{1}(\omega) \boldsymbol{v}+k^{2} A_{2}(\omega) \boldsymbol{v}+k^{3} A_{3}(\omega) \boldsymbol{v}+k^{4} A_{4}(\omega) \boldsymbol{v}=0
$$

The negative of the imaginary part of $k,-k_{i}$, is the spatial amplification rate, whereas its real part, $k_{r}$, corresponds to the streamwise wavenumber of the travelling wave, whose frequency is given by $\omega$. 
The solution of the equation (5.1) leads to a blow-up of spurious eigenvalues due to numerical discretization, which makes it difficult to detect the correct eigenvalue with physical significance. However, unstable waves propagating downstream are characterized by a positive axial phase velocity, $c_{\phi}=\omega / k_{r}$, and a negative growth rate, $k_{i}$. Thus, for positive $\omega$ the eigenvalues of interest are located in the fourth quadrant, i.e. with $k_{r}>0$ and $k_{i}<0$. To overcome issues related to detection of the correct eigenvalue among many spurious ones, the solution of (5.1) is evaluated by searching for the eigenvalue closest to the one predicted via the Gaster transformation. The Gaster relation allows us to relate the temporal growth rate to the spatial growth rate in the vicinity of marginal stability: see Gaster (1962). In fact, the same dispersion relation is considered for the temporal analysis and spatial analysis, but it is solved for different variables. On the neutral curves temporal and spatial results are coincident, since $k_{i}=\omega_{i}=0$ and $(k, \omega) \in \mathbb{R}$. For small imaginary parts a Taylor series expansion in the neighbourhood of the neutral curves is used. The Gaster relation is

$$
\omega_{i}^{(T)}=-c_{g} k_{i}^{(S)}
$$

which states that in the limit of small imaginary parts the temporal growth rate, $\omega_{i}^{(T)}$, and the spatial growth rate, $-k_{i}^{(S)}$, are related by the group velocity, $c_{g}$. Therefore, an approximation of the spatial instability results can be obtained from the temporal analysis via the Gaster transformation.

For the spatial stability analysis, growth rates $-k_{i}\left(\omega_{r}, m\right)$ of the unstable spatial modes are represented as a function of the non-dimensional frequency, $\omega_{r}$, for different $m$ and downstream locations. In figures 10 and 11 the dashed lines represent the results of the Gaster approximation, whereas the solid lines correspond to the values $-k_{i}$ obtained from the spatial stability analysis. For downstream locations $x / d \geqslant 1.75$ the dashed and solid lines in figures 10 and 11 are practically coincident; in fact, the Gaster transformation and the spatial stability analysis produce roughly the same results, despite the large values of the spatial growth rate, $-k_{i}$. For the downstream location $x / d=0.5$ the Gaster relation significantly underestimates the growth rate obtained with the spatial analysis. This discrepancy is mainly due to the large values of $k_{i}$ and to the strong wake velocity deficit observed at this downstream location. Indeed, the Gaster transformation produces more accurate results for convectively dominated flows: see e.g. Olendraru et al. (1999), Schmid \& Henningson (2001) and Olendraru \& Sellier (2002). Furthermore, as mentioned above, it has been verified that the strong peak of the spatial growth rate for $m=1$ at $x / d=0.5$, obtained from the spatial stability analysis, is not related to an absolute instability; indeed, no intersection in the complex plane $k_{r}-k_{i}$ between the positive and negative branches, $k^{+}$and $k^{-}$, was detected; see Huerre (1998), Olendraru et al. (1999), Schmid \& Henningson (2001) and Olendraru \& Sellier (2002) for details.

The results obtained with the spatial stability analysis are qualitatively similar to those obtained with the temporal stability analysis presented in $\S 4$. By considering the low-frequency instability connected to the hub vortex, indicated in figures 10 and 11 by a vertical dashed line corresponding to $\omega_{r}=3.18$, it is evident that for downstream locations from $x / d=0.5$ up to $x / d=2.75$ the selected unstable mode is the one with $m=1$. Further downstream, i.e. at $x / d \approx 3$, the most unstable mode switches to $m=2$ but with a much smaller spatial growth rate. At $x / d=4$ the selected mode is the one with $m=4$.

In order to determine the dominant unstable mode related to the low-frequency instability of the hub vortex, the growth rates corresponding to $\omega_{r}=3.18$ for the 

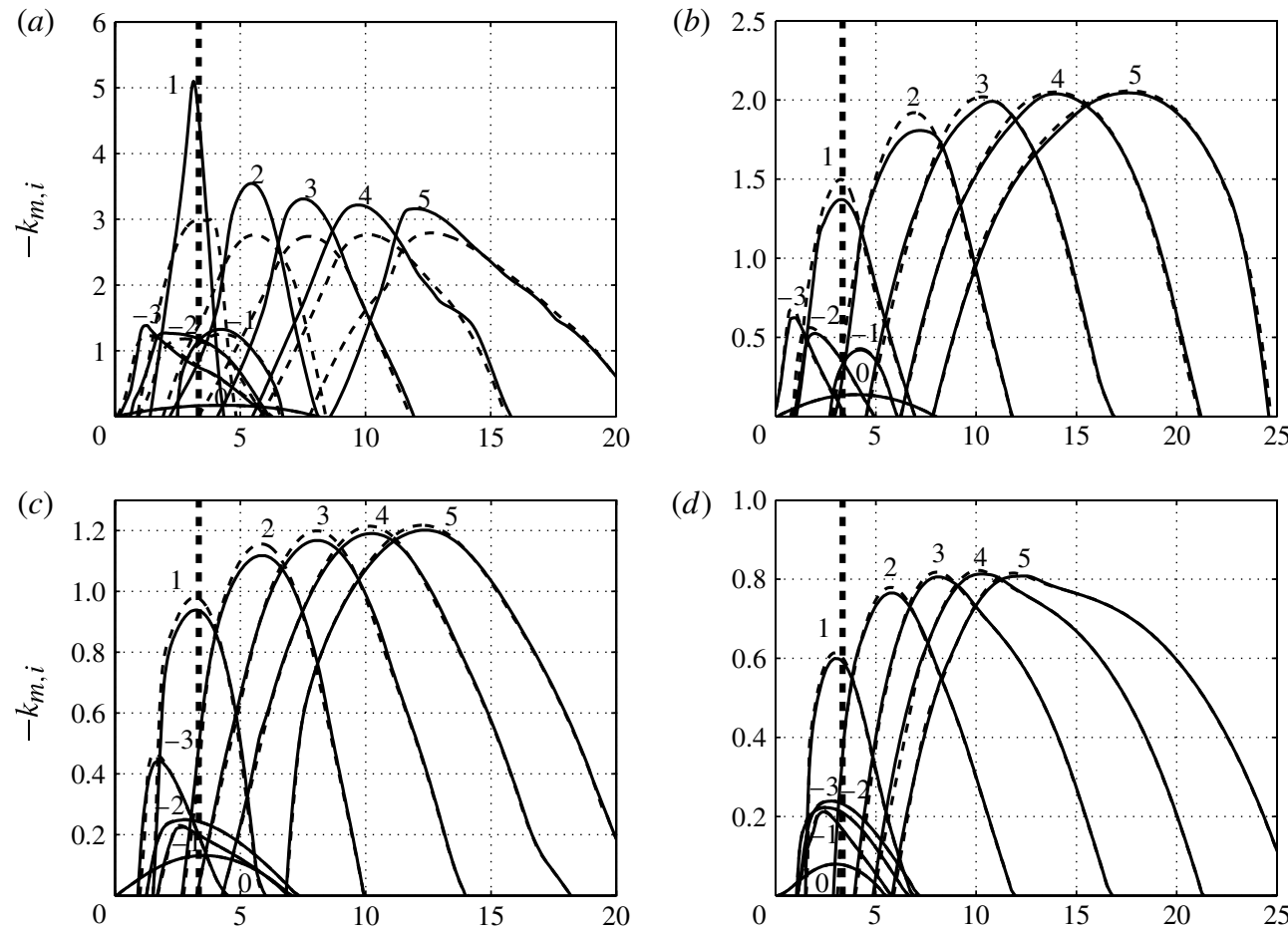

(d)
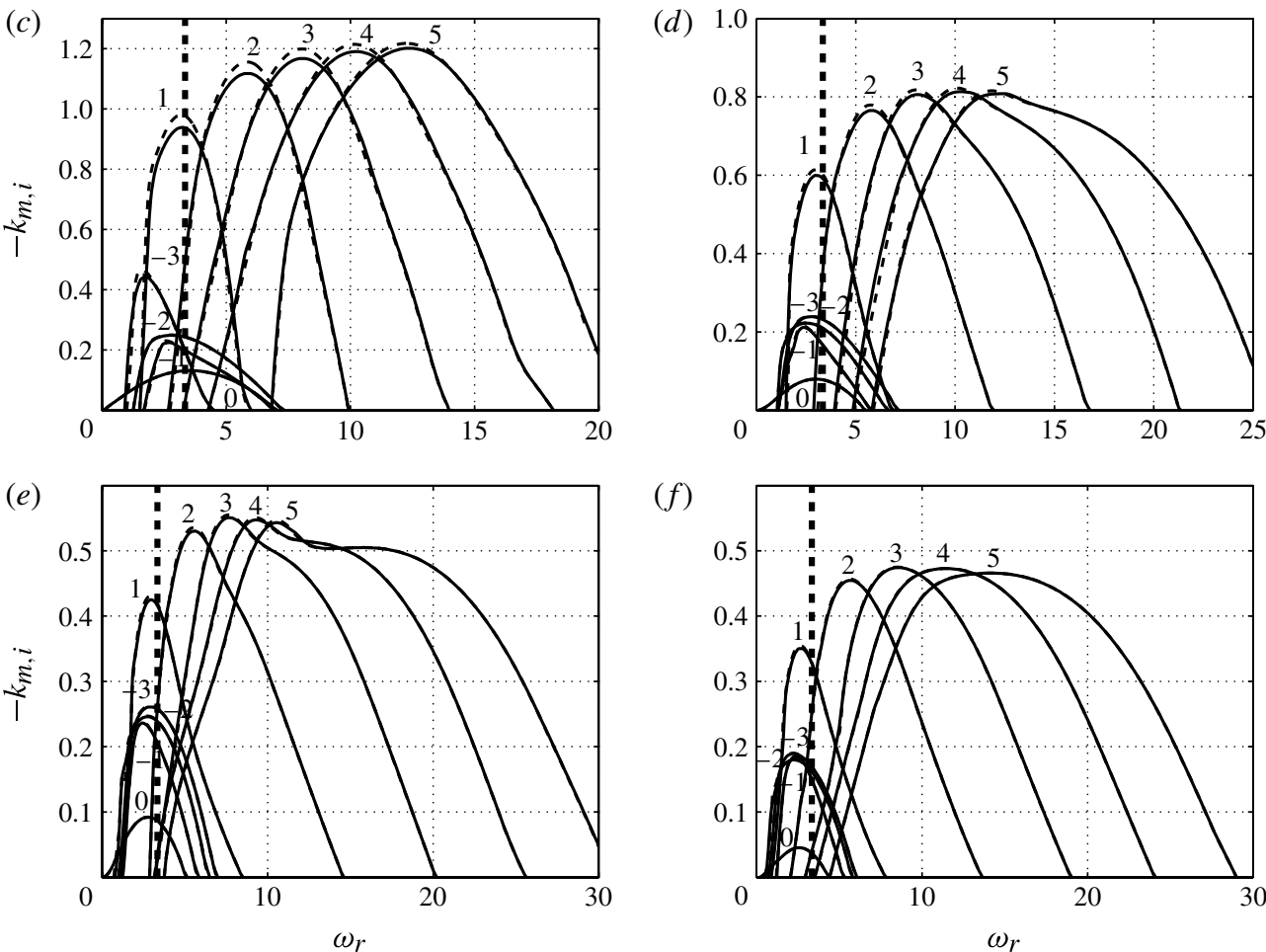

$(f)$

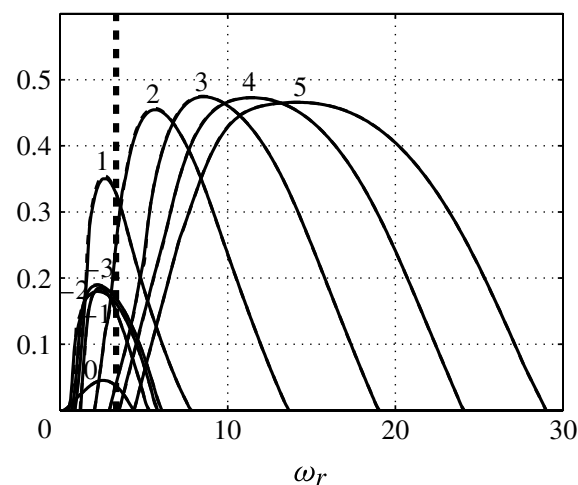

FIGURE 10. Growth rate evaluated via the Gaster approximation (dashed line) and spatial stability analysis (solid line) as a function of the non-dimensional frequency for downstream locations from $x / d=0.5$ up to $x / d=1.75$. The vertical dashed line corresponds to the non-dimensional frequency measured experimentally and related to the hub vortex instability: (a) $x / d=0.5 ;(b) x / d=0.75 ;(c) x / d=1 ;(d) x / d=1.25 ;(e) x / d=1.5 ;(f) x / d=1.75$.

modes with $m=1$ and $m=2$ are plotted in figure 12 as a function of the streamwise location. Then, the integral amplification factor is evaluated for the two modes $m=1$ and $m=2$, as proposed by Oberleithner et al. (2011) and Juniper, Tammisola \& Lundell (2011), according to

$$
G(\omega, m)=\exp \left(\int_{X_{0}}^{X_{S}}-k_{i}\left(\omega, m, X^{\prime}\right) \mathrm{d} X^{\prime}\right),
$$




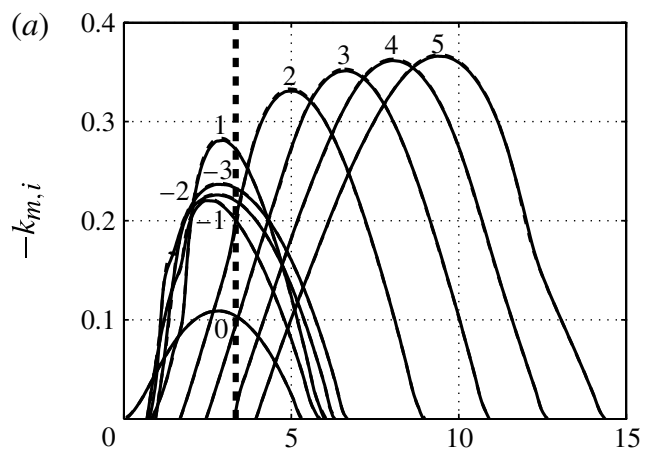

(b)
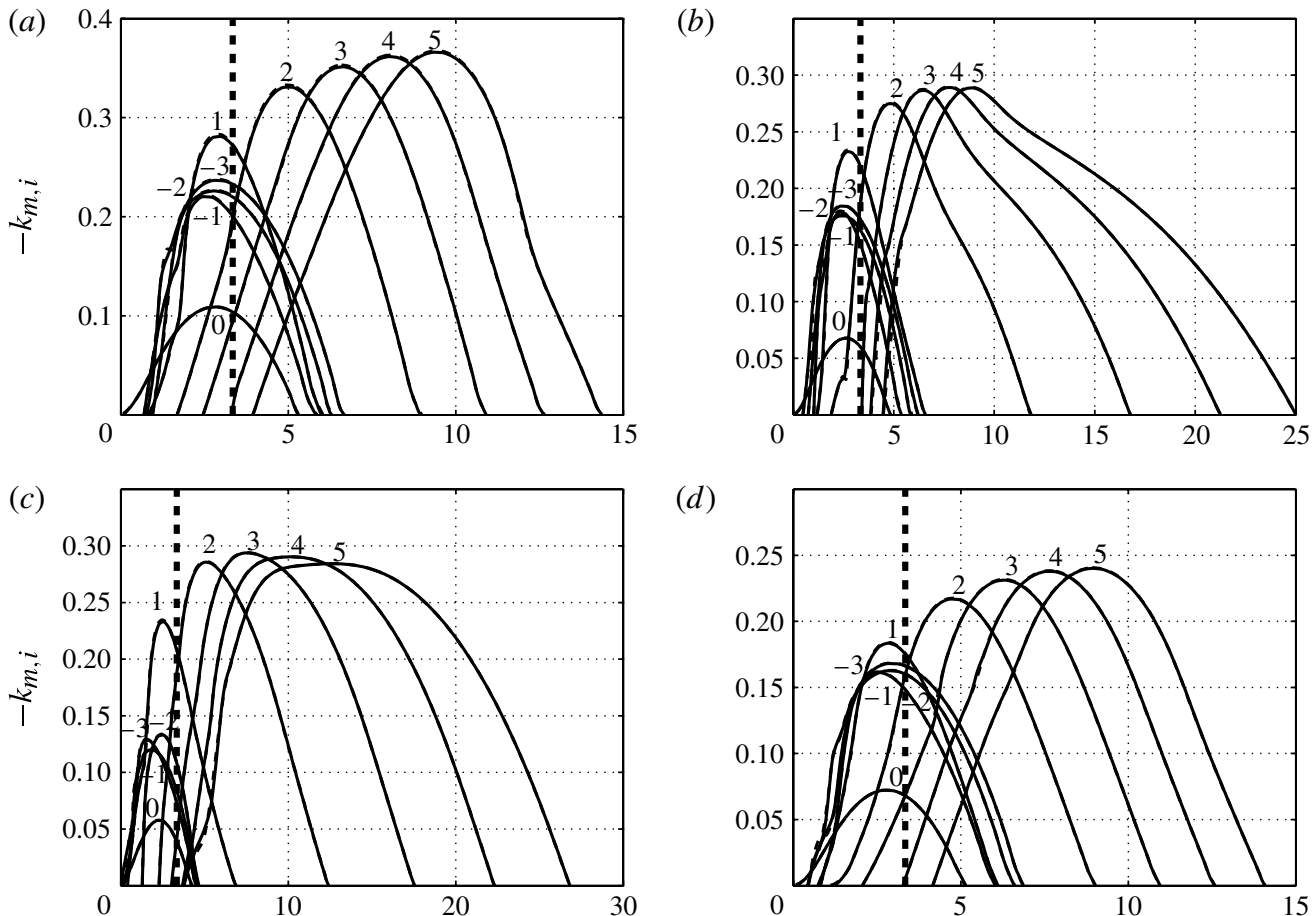

(d)
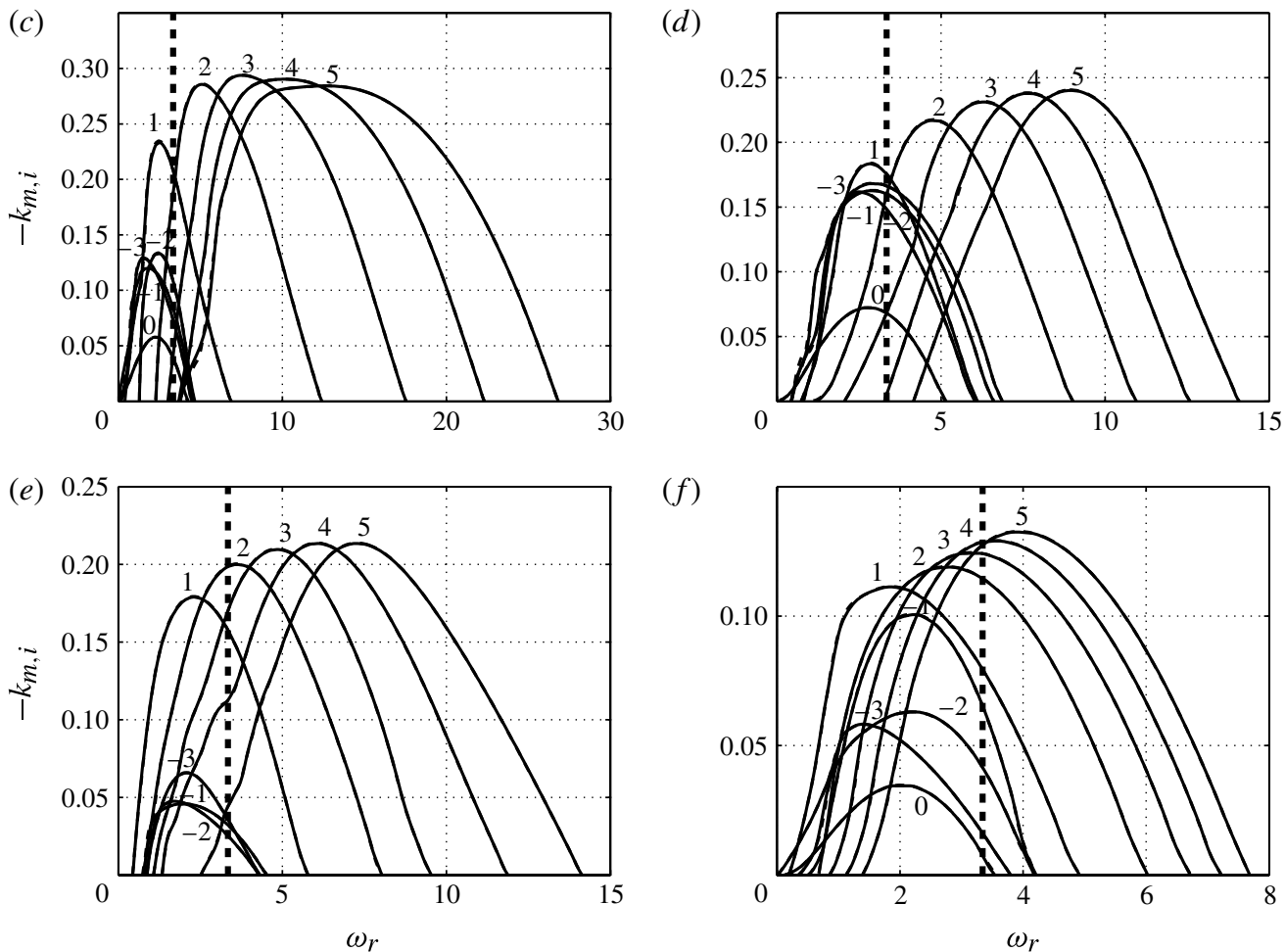

$(f)$

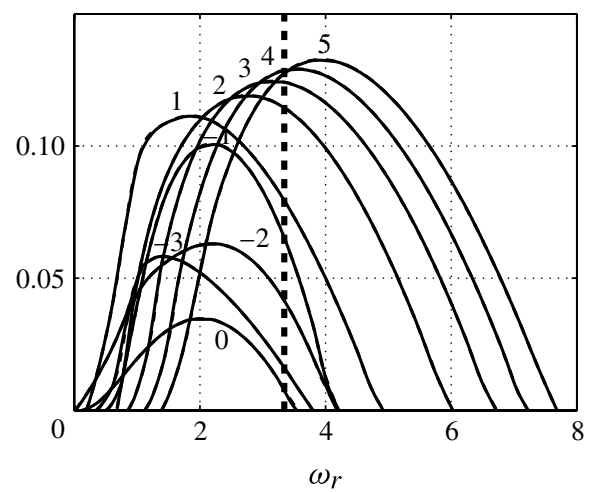

FIGURE 11. Growth rate evaluated via the Gaster approximation (dashed line) and spatial stability analysis (solid line) as a function of the non-dimensional frequency for downstream locations from $x / d=2$ up to $x / d=4$. The vertical dashed line corresponds to the nondimensional frequency measured experimentally and related to the hub vortex instability: (a) $x / d=2 ;(b) x / d=2.25 ;(c) x / d=2.5 ;(d) x / d=2.75 ;(e) x / d=3 ;(f) x / d=4$.

where $X_{S}$ is the streamwise position where the flow re-enters in a stable domain, and $G(\omega, m)$ represents the overall amplification of the mode $m$ at the frequency $\omega$ within the wake, after it has gone through its entire amplification process. This integral amplification factor, computed from the position $x / d=0.5$ up to $x / d=4$, is equal to 6.6 for the unstable mode with $m=1$, and for the mode with $m=2$ it is 2.47 , thereby suggesting the predominance of the $m=1$ mode, i.e. a counter-winding singlehelix structure. Using this simple procedure, the integral amplification factor can be 


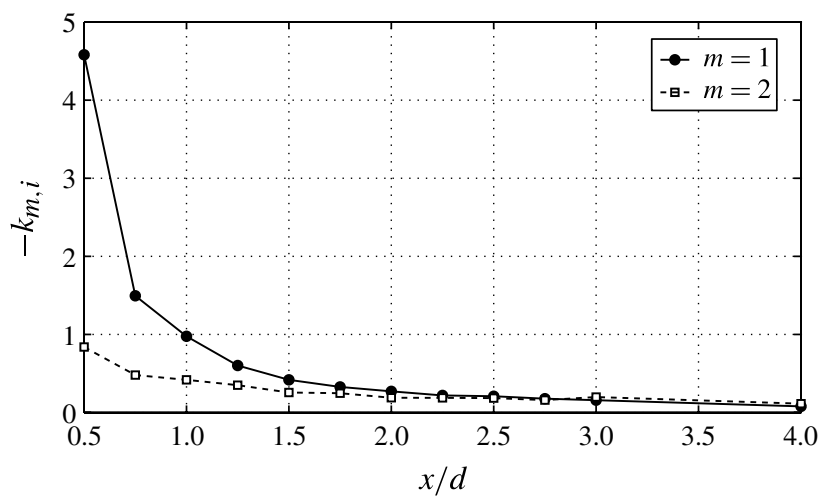

FIGURE 12. Spatial growth rates of the unstable modes with $m=1$ and $m=2$ as a function of the downstream location, $x$, corresponding to the non-dimensional frequency $\omega_{r}=3.18$.

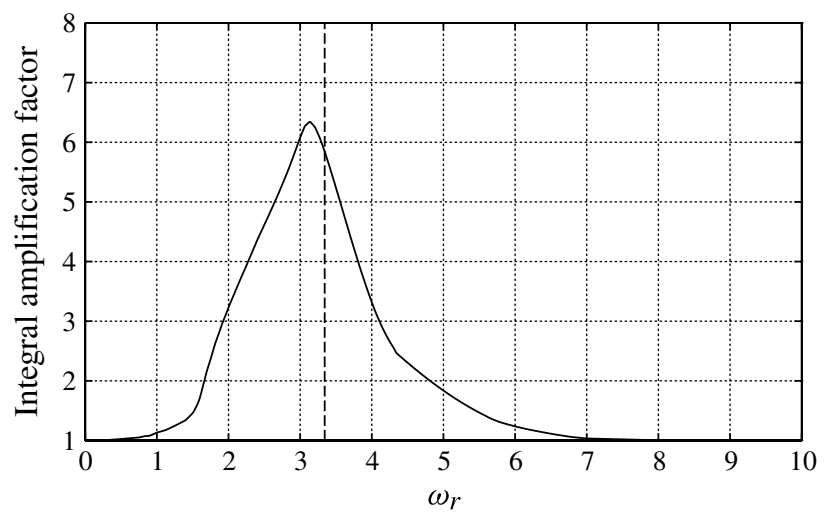

FIGURE 13. Integral amplification factor of the unstable mode with $m=1$ as a function of the pulsation $\omega_{r}$.

systematically determined as a function of the frequency for the single helical mode, as shown in figure 13. It is striking to observe that the counter-winding single-helix mode is not only the most amplified in this frequency range, but it reaches its maximum amplification at a frequency very close to the one observed experimentally. While the present analysis does not reveal the origin of the sharp frequency selection mechanism observed in the experiments, this shows that the most amplified frequency downstream from $x / d=0.5$ matches the one measured in our experiments.

With the spatial stability analysis, the real part of $k, k_{r}$, represents the axial wavenumber of the respective unstable mode. In figure $14, k_{r}$ is plotted as a function of the non-dimensional frequency, $\omega_{r}$, for several downstream locations, considering different values of $m$. For the selected mode with $m=1$ representing the hub vortex instability, the axial growth rate is $\sim 4.5$ and it is slightly reduced by moving downstream, except for the position $x / d=0.5$ where $k_{r}=6.31$ is obtained. Therefore, the single-helix instability of the hub vortex is characterized by an axial wavelength, $\lambda / d=2 \pi / k_{r}$, of $\sim 1$ at $x / d=0.5$, and then it is increased by moving downstream with a value of 1.65 at the location $x / d=4$. 

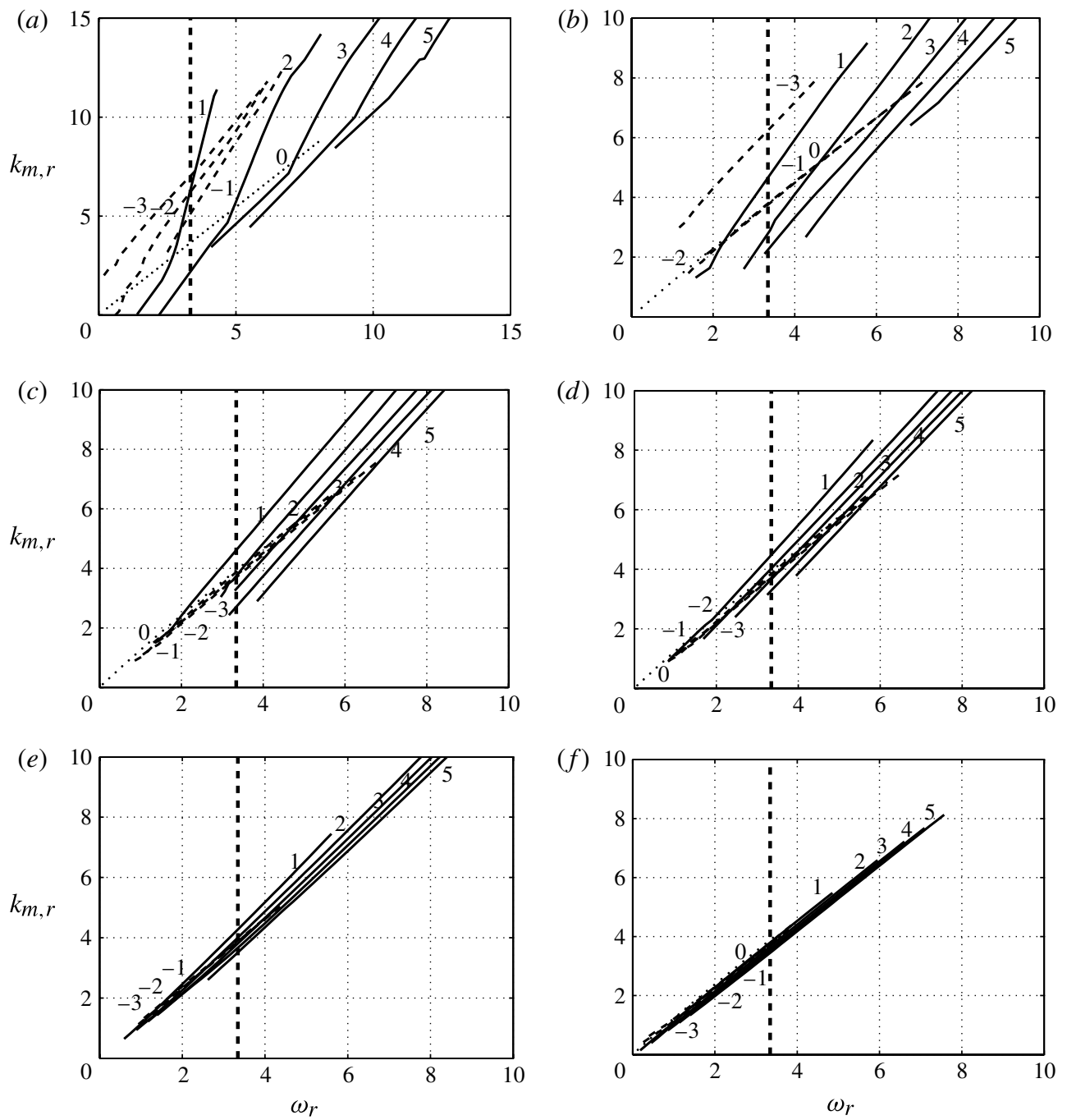

FIgURE 14. Axial wavenumber, $k_{r}$, evaluated via the spatial stability analysis as a function of the non-dimensional frequency. The vertical dashed line corresponds to the nondimensional frequency measured experimentally and related to the hub vortex instability: (a) $x / d=0.5 ;$ (b) $x / d=1 ;(c) x / d=1.5 ;(d) x / d=2 ;(e) x / d=3 ;(f) x / d=4$.

It is now possible to perform the reconstruction of the single helix global mode representing the hub vortex instability by integrating the unstable eigenmode $m=1$ in the $x$-direction, in agreement with (5.4) (see Oberleithner et al. 2011; Juniper et al. 2011):

$$
\boldsymbol{u}(x, r, \theta, t) \approx \operatorname{Re}\left\{A_{0}(X) \hat{\boldsymbol{u}}(r, X) \exp \left[i\left(\int_{X_{0}}^{X} k\left(X^{\prime}, \omega\right) \mathrm{d} X^{\prime}+m \theta-\omega t\right)\right]\right\}
$$

where $k$ is the complex wavenumber, $\omega$ is the instability frequency and $\hat{u}$ gives the eigenmodes at the $X$-position normalized using the Euclidean norm, and phase-aligned at the characteristic vortex radius. The slowly varying amplitude $A_{0}(X)$ could be 
considered uniform to first order, in agreement with the WKBJ formulation. The axial vorticity of the local unstable mode with $m=1$ is plotted in figure 15 . These vorticity structures clearly represent a coherent evolution of a single-helix unstable mode, although these eigenfunctions were obtained from experimental data acquired at different downstream locations. Finally, the global unstable mode with $m=1$ is reconstructed along the $x$-direction, as for the axial vorticity reported in figure 16 .

Further wind tunnel measurements were performed in order to provide experimental evidence of this outlined helicoidal mode structure, and in particular to assess the axial wavenumber predicted by the local stability analysis. To this end, simultaneous measurements with two single-component hot-wire anemometers were carried out. For these tests one probe was placed at the fixed location $x / d=0.13, y / d=0.1, z / d=0$ and another probe was set on the traversing system, which allows displacements of the probe along the $x$-direction, and at the transversal position $y / d=-0.1$ and $z / d=0$. In other words the two probes were symmetrically located with respect to the wake centre and at a transversal distance where the spectral component related to the hub vortex instability was typically detected with maximum energy. For each acquired velocity signal, the spectral component of interest was extracted by using a filtering technique based on proper orthogonal decomposition proposed in Iungo \& Lombardi (2011), with a frequency resolution of $5 \mathrm{~Hz}$. This technique presents the advantage in automatically detecting and extracting the dominant spectral component, thus emphasizing the correlation between simultaneous signals by removing experimental noise and other spectral contributions.

The cross-correlation coefficient between the extracted components from the velocity signals acquired simultaneously on the two sides of the wake was then calculated for different downstream locations. These tests were driven by the idea that when the hub vortex is characterized by helicoidal instability, the cross-correlation coefficient between the time series acquired on the two sides of the wake should follow a harmonic function when one probe is placed at a fixed position and the other one is moved downstream. Furthermore, the wavelength of the harmonic function detected through the cross-correlation coefficient should reproduce the same axial wavenumber as the helicoidal structure. Indeed, the cross-correlation coefficient evaluated for different downstream locations clearly reproduces a harmonic trend, as shown in figure 17, with a slightly reducing amplitude and increasing wavelength by moving downstream, which can be connected to the diffusion of the hub vortex. Therefore, the simultaneous hot-wire measurements confirm the presence of a helicoidal instability of the hub vortex predicted by the linear stability analysis.

Moreover, the instantaneous wavelength of the cross-correlation coefficient as a function of the downstream location is then computed via the Hilbert transform: see Iungo \& Lombardi (2011) for details. This parameter is plotted in figure 18 and compared to the results obtained with the spatial stability analysis, i.e. with $2 \pi / k_{r}$ corresponding to the unstable mode with $m=1$ and at the non-dimensional frequency corresponding to the hub vortex instability, $\omega_{r}=3.18$. The experimental data generally confirm the results obtained with the linear stability analysis, i.e. the hub vortex is characterized by a helicoidal instability with a wavelength $\lambda / d=1$ at the downstream location $x / d=0.5$. Then, it is gradually increased by moving downstream up to a value $\lambda / d \approx 1.6$ at $x / d=4$. Slightly different results are found for the locations $0.5<x / d<1.5$, where the wavelength varies more rapidly. However, both experimental data and linear instability results show good agreement and a gradual increase of the streamwise wavelength with increasing streamwise distance from the turbine location. By moving downstream, an increase of the wavelength 


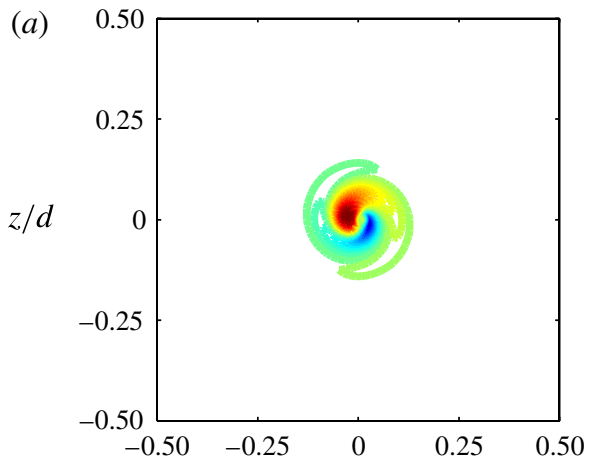

(b)

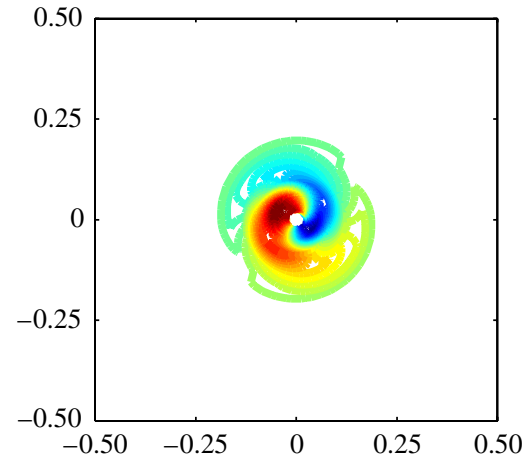

(c)

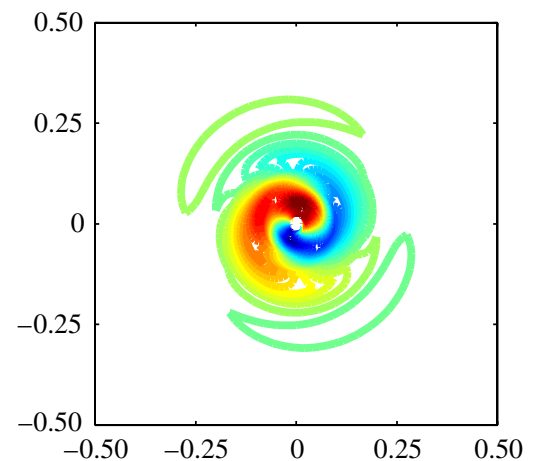

(e)

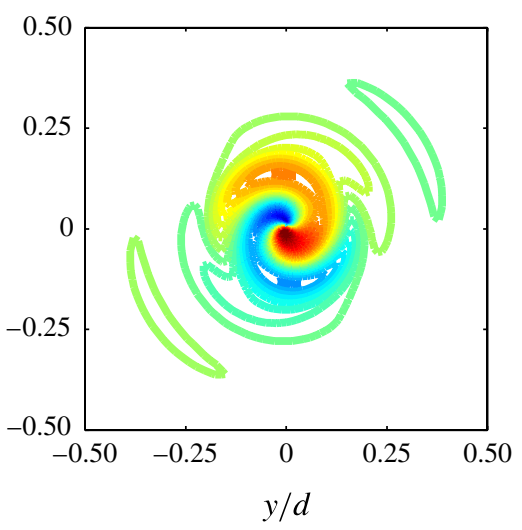

(d)

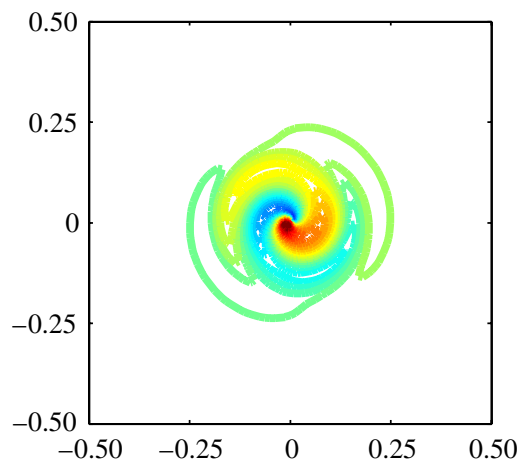

$(f)$

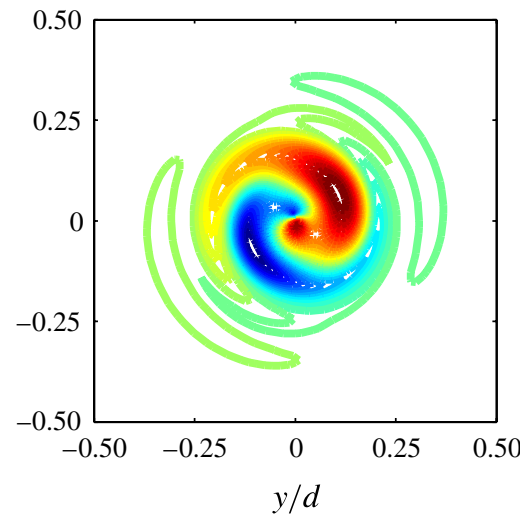

FIGURE 15. Axial vorticity of the selected unstable eigenmode with $m=1$ reconstructed via the spatial stability analysis: (a) $x / d=0.5 ;(b) x / d=0.75 ;(c) x / d=1 ;(d) x / d=1.5 ;(e)$ $x / d=2 ;(f) x / d=3$.

of the unstable mode related to the hub vortex is connected to the diffusion of the hub vortex and the imminent breakdown of this vorticity structure, which compares well with previous works such as those of Sarpkaya (1971) and Felli et al. (2011). Furthermore, from figure 17, and also from the Fourier spectra shown in figure 4, 


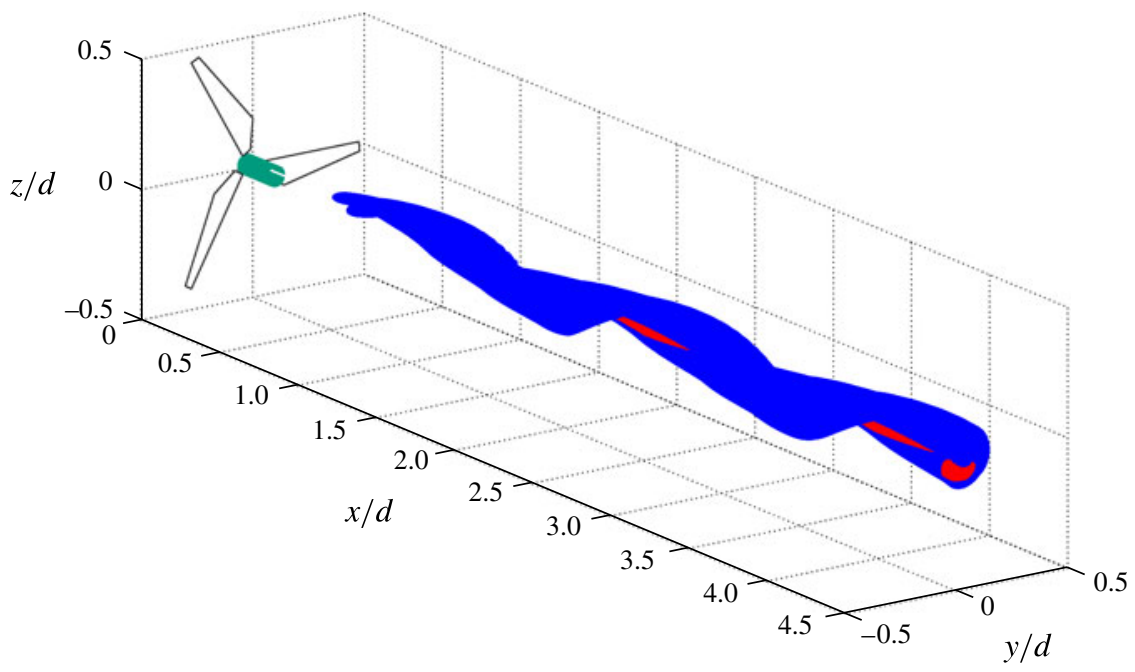

FIGURE 16. Reconstruction of the hub vortex instability corresponding to the mode with $m=1$. Iso-surface of the axial vorticity.

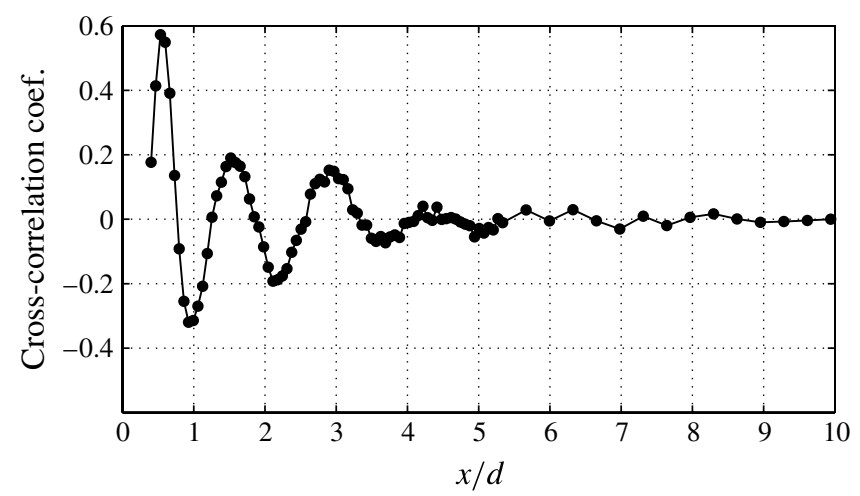

FIGURE 17. Cross-correlation coefficient between hot-wire time-series acquired simultaneously at $y / d= \pm 0.1$ and $z / d=0$ as a function of the streamwise location.

the energy content connected to the hub vortex instability decays in the downstream direction, whereas the linear stability analysis predicts growth. This feature can be ascribed to the linearity of the stability analysis, which does not take into account the nonlinear saturation of the mode. Indeed, diffusive effects caused by Reynolds stresses are neglected, which affects the estimated growth rates.

\section{Discussion and final remarks}

Wind tunnel measurements of a wake produced by a wind turbine immersed in uniform flow showed the presence of an axial vorticity structure in the very near wake, which is denoted as the hub vortex. The hub vortex is characterized by oscillations with a frequency equal to 0.34 times the rotational frequency of the wind turbine rotor. In previous works these flow fluctuations were ascribed to wake wandering or meandering, which consists of transversal oscillations of the wind turbine wake. Wake 


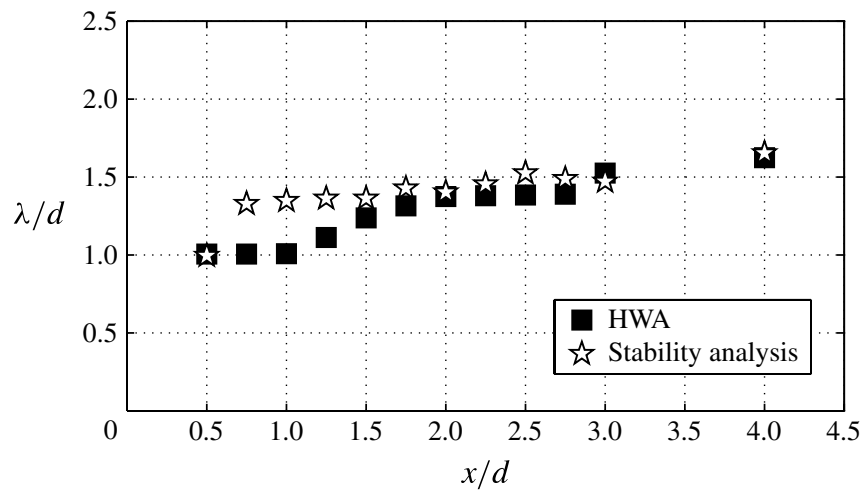

FIGURE 18. Comparison of the axial wavelength of the global unstable mode evaluated via the linear stability analysis and the simultaneous hot-wire measurements, HWA.

meandering was investigated in detail by Medici \& Alfredsson (2008); in fact, these authors stated that this phenomenon is mainly excited by the shedding of vorticity structures from the rotor disc acting as a bluff body.

To further investigate the above-mentioned low-frequency wake instability detected experimentally, linear stability analysis was performed. The evolution of perturbations acting on a mean flow obtained using wind tunnel velocity measurements was investigated. The temporal stability analysis showed that the most unstable mode corresponding to the low-frequency instability of the hub vortex is characterized by a single-helix counter-winding mode, rotating in the same direction as the hub vortex. This result is obtained in the near wake up to a downstream distance of 2.5 rotor diameters. Further downstream, unstable modes with a higher azimuthal wavenumber, $m$, start to be dominant. However, by moving downstream, a reduction of the growth rates of the unstable modes is generally observed. Therefore, possible competition between different unstable modes can take place by moving downstream, while the hub vortex is rapidly diffusing. It should be pointed out that the single-helix counterwinding unstable mode $(m=1)$ is not always the one with the highest growth rate, but it is generally the one corresponding to the experimentally measured frequency of the spectral component connected to the hub vortex instability. This suggests that for the wind turbine wake flow produced in the wind tunnel, unstable modes with a higher azimuthal wavenumber are damped, and thus the dominating mode turns out to be the one with $m=1$. This reduction of the growth rates related to unstable modes with a higher azimuthal wavenumber could be due to diffusive effects connected to turbulent fluctuations, which are not taken into account in the linear stability analysis.

In order to investigate in greater depth possible competition between unstable modes with a different azimuthal wavenumber, $m$, a spatial stability analysis was performed, which is more suitable for convectively unstable flows. The spatial stability analysis confirmed the results obtained from the temporal analysis, and in addition it allowed us to determine by means of evaluation of the integral growth rate that the dominant unstable mode has an azimuthal wavenumber equal to one $(m=1)$. The global unstable mode characterized by a single-helix counter-winding structure has been reconstructed, in particular characterizing the evolution of the axial wavelength by moving downstream. Further hot-wire measurements were then performed to assess experimentally the presence of helicoidal instability of the hub vortex. To this end, 
simultaneous hot-wire measurements were performed by placing two probes at hub height, on both sides of the wake and at the same spanwise distance from the hub, i.e. where the frequency connected to the hub vortex instability has the maximum energy. During the tests one probe was placed at a fixed location, while the other one was moved downstream. The spectral component connected to the hub vortex instability was then extracted from the velocity signals. The cross-correlation coefficient between the signals acquired simultaneously, evaluated as a function of the streamwise location, has shown a harmonic trend confirming the presence of helicoidal instability of the hub vortex. Furthermore, the local axial wavelength of this harmonic function obtained from the cross-correlation coefficient accurately confirms the evolution of the axial wavenumber of the most unstable mode predicted through the linear stability analysis.

The single-helix counter-winding unstable mode of the hub vortex obtained by this linear stability analysis is in good agreement with the instability of the hub vortex produced by a marine propeller visualized in Felli et al. (2011). The main difference in comparison to the present work is that for the marine propeller the frequency related to hub vortex instability is equal to the rotational frequency of the rotor, whereas for the present experimental case this frequency is 0.34 times the rotational frequency of the rotor, which is in good agreement with previous wind tunnel investigations of wind turbine models, e.g. Medici \& Alfredsson (2008), Chamorro \& Porté-Agel (2010) and Zhang et al. (2012).

While it is very tempting to attribute the sharp frequency selection typical of oscillator behaviour to an absolutely unstable region in the near-wake region, this could not be confirmed experimentally with the measurement techniques used at present. Nevertheless, our study has revealed the following.

(i) The most amplified frequency downstream of $x / d=0.5$ matches the frequency measured in our experiments. This might be pure coincidence, or it might indicate a selective noise amplification mechanism yet to be identified.

(ii) In contrast to most commonly accepted nonlinear global mode shapes predicted from nonlinear front theories (see Chomaz 2005 for a review and Couairon \& Chomaz 1999 for a detailed analysis), the coherent structure observed in this study does not display a sharp front located at the convective-to-absolute transition. The spectral energy associated with the hub vortex is seen to increase up to $x / d=1$ before it starts to decay, as is readily seen in figure 4 . This observation is consistent with the study of Felli et al. (2011), who have also observed that hub vortex instability only starts at a downstream distance of a few rotor diameters.

Therefore, unravelling the detailed origin of the sharp frequency selection by means of precise measurements in the very near-wake region constitutes a natural continuation of this study, as well as a formidable experimental challenge.

Further wind tunnel tests and respective linear instability analyses are currently underway in order to address more practical applications, such as the case of a wind turbine immersed in a boundary layer flow, or the case of interaction of wind turbine wakes within a wind farm.

\section{REFERENCES}

Alfredsson, P. H. \& DAhlberg, J. A. 1979 A preliminary wind tunnel study of windmill wake dispersion in various flow conditions. Technical Note AU-1499, Part 7, FFA, Stockholm, Sweden.

Antkowiak, A. 2005 Short-term dynamics of an isolated vortex. PhD thesis, IMFT-Université Paul Sabatier. 
BARKLEY, D. 2006 Linear analysis of the cylinder wake mean flow. Europhys. Lett. 37 (5), $750-756$.

Burton, T., Sharpe, D., Jenkins, N. \& Bossanyi, N. 2001 Wind Energy Handbook. Wiley.

Camarri, S., Fallenius, B. E. G. \& Fransson, J. H. M. 2013 Stability analysis of experimental flow fields behind a porous cylinder for the investigation of the large-scale wake vortices. J. Fluid Mech. 715, 499-536.

Canuto, C., Hussaini, M. Y., Quarteroni, A. \& Zang, T. A. 1993 Spectral Methods in Fluid Dynamics. Springer.

Chamorro, L. P. \& Porté-Agel, F. 2009 A wind-tunnel investigation of wind-turbine wakes: boundary-layer turbulence effects. Boundary-Layer Meteorol. 132 (1), 129-149.

Chamorro, L. P. \& Porté-Agel, F. 2010 Effects of thermal stability and incoming boundarylayer flow characteristics on wind-turbine wakes: a wind-tunnel study. Boundary-Layer Meteorol. 136 (3), 515-533.

Chomaz, J.-M. 2005 Global instabilities in spatially developing flows: non-normality and nonlinearity. Аnnu. Rev. Fluid Mech. 37, 357-392.

Couniron, A. \& Chomaz, J.-M. 1999 Fully nonlinear global modes in slowly varying flows. Phys. Fluids 11 (12), 3688-3703.

Felli, M., CAmussi, R. \& DiFelice, F. 2011 Mechanisms of evolution of the propeller wake in the transition and far fields. J. Fluid Mech. 682, 5-53.

Gallaire, F. \& Chomaz, J. M. 2003 Mode selection in swirling jet experiments: a linear stability analysis. J. Fluid Mech. 494, 222-224.

GASTER, M. 1962 A note on the relation between temporally-increasing and spatially-increasing disturbances in hydrodynamic instability. J. Fluid Mech. 14, 222-224.

GuptA, B. P. \& Loewy, R. G. 1974 Theoretical analysis of the aerodynamic stability of multiple, interdigitated helical vortices. AIAA J. 12, 1381-1387.

Huerre, P. 1998 Hydrodynamic Instabilities in Open Flows. Cambridge University Press.

IUNGO, G. V. \& LOMBARDI, E. 2011 A procedure based on proper orthogonal decomposition for time-frequency analysis of time series. Exp. Fluids 51, 969-985.

IVAnell, S., Mikkelsen, R., SøRensen, J. N. \& Henningson, D. 2010 Stability analysis of the tip vortices of a wind turbine. Wind Energy 13, 705-715.

IVAnell, S., Sørensen, J. N., Mikkelsen, R. \& Henningson, D. 2009 Analysis of numerically generated wake structures. Wind Energy 12, 63-80.

Joukowski, N. E. 1912 Vortex theory of a rowing screw. Trudy Otdeleniya Fizicheskikh Nauk Obshchestva Lubitelei Estestvoznaniya 16 (1)in Russian.

Juniper, M. P., TAmmisola, O. \& Lundell, F. 2011 The local and global stability of confined planar wakes at intermediate Reynolds number. J. Fluid Mech. 686, 218-238.

Kerswell, R. R. \& DAVEY, A. 1996 On the linear instability of elliptic pipe flow. J. Fluid Mech. 316, 307-324.

Klein, R., Majda, A. J. \& Damodaran, K. 1995 Simplified equations for the interaction of nearly parallel vortex filaments. J. Fluid Mech. 288, 201-248.

Leibovich, S. \& Stewartson, K. 1983 A sufficient condition for the instability of columnar vortices. J. Fluid Mech. 126, 335-356.

Leontini, J. S., Thompson, M. C. \& Hourigan, K. 2010 A numerical study of global frequency selection in the time-mean wake of a circular cylinder. J. Fluid Mech. 645, 435-446.

Levy, H. \& Forsdyke, A. G. 1928 The steady motion and stability of a helical vortex. Proc. R. Soc. Lond. A 120, 670-690.

Lighthill, J. 1978 Waves in Fluids. Cambridge University Press.

Lu, H. \& Porté-Agel, F. 2011 Large-eddy simulation of a very large wind farm in a stable atmospheric boundary layer. Phys. Fluids 23 (6), 065101.

Massouh, F. \& DobReV, I. 2007 Exploration of the vortex wake behind of wind turbine rotor. J. Phys.: Conf. Ser. 75012036.

Medici, D. \& Alfredsson, P. H. 2006 Mesurements on a wind turbine wake: 3D effects and bluff body vortex shedding. Wind Energy 9 (3), 219-236. 
Medici, D. \& Alfredsson, P. H. 2008 Measurements behind model wind turbines: further evidence of wake meandering. Wind Energy 11, 211-217.

Meliga, P., Sipp, D. \& ChomaZ, J.-M. 2009 Elephant modes and low frequency unsteadiness in a high Reynolds number, transonic afterbody wake. Phys. Fluids 21 (5), 054105.

Monkewitz, P. A. 1988 A note on vortex shedding from axisymmetric bluff bodies. J. Fluid Mech. 192, 561-575.

Oberleithner, K., Sieber, M., Nayeri, C. N., Paschereit, C. O., Petz, C., Hege, H.-C., NOACK, B. R. \& WYGNANSKI, I. 2011 Three-dimensional coherent structures in a swirling jet undergoing vortex breakdown: stability analysis and empirical mode construction. J. Fluid Mech. 679, 383-414.

OKulov, V. L. 2004 On the stability of multiple helical vortices. J. Fluid Mech. 521, 319-342.

OKulov, V. L. \& Sørensen, J. N. 2007 Stability of helical tip vortices in a rotor far wake. J. Fluid Mech. 576, 1-25.

Olendraru, C. \& Sellier, A. 2002 Viscous effects in the absolute/convective instability of the Batchelor vortex. J. Fluid Mech. 459, 371-396.

Olendraru, C., Sellier, A., Rossi, M. \& Huerre, P. 1999 Inviscid instability of the Batchelor vortex: absolute-convective transition and spatial branches. Phys. Fluids 11, 1805-1820.

Ortega, J. M., Bristol, R. L. \& SAVAs, Ö. 2003 Experimental study of the instability of unequal-strength counter-rotating vortex pairs. J. Fluid Mech. 474, 35-84.

Pier, B. \& Huerre, P. 2001 Nonlinear synchronization in open flows. J. Fluids Struct. 15, $471-480$.

Porté-Agel, F., Wu, Y-T., Lu, H. \& Conzemius, R. J. 2011 Large-eddy simulation of atmospheric boundary layer flow through wind turbines and wind farms. J. Wind Engng Ind. Aerodyn. 99 (4), 154-168.

SARPKAYA, T. 1971 On stationary and travelling vortex breakdowns. J. Fluid Mech. 45, 545-559.

SCHITO, P. 2012 Large eddy simulations of wind turbines: interaction with turbulent flow. PhD thesis, Department of Mechanical Engineering, Politecnico di Milano.

Schmid, P. J. \& Henningson, D. S. 2001 Stability and Transition in Shear Flows. Springer.

Sherry, M., Sheridan, J. \& LoJacono, D. 2010 Horizontal axis wind turbine tip and root vortex measurements. In 15th International Symposium on Applications of Laser Techniques to Fluid Mechanics.

SiPP, D. \& LEBEDEV, A. 2007 Global stability of base and mean flows: a general approach and its applications to cylinder and open cavity flows. J. Fluid Mech. 593, 333-358.

Sørensen, J. N. 2011 Instability of helical tip vortices in rotor wakes. J. Fluid Mech. 682, 1-4.

Vermeer, L. J., Sørensen, J. N. \& Crespo, A. 2003 Wind turbine wake aerodynamics. Prog. Aerosp. Sci. 39 (6/7), 467-510.

Whale, J., Papadopoulos, K. H., Anderson, C. G., Helmis, C. G. \& Skyner, D. J. 1996 A study of the near wake structure of a wind turbine comparing measurements from laboratory and full-scale experiments. Solar Energy 56 (6), 621-633.

Widnall, S. E. 1972 The stability of a helical vortex filament. J. Fluid Mech. 54 (4), 641-663.

Zhang, W., MArkfort, C. D. \& Porté-Agel, F. 2012 Near-wake flow structure downwind of a wind turbine in a turbulent boundary layer. Exp. Fluids 52 (5), 1219-1235. 